ARTICLE

\title{
Circuit-specific hippocampal $\Delta$ FosB underlies resilience to stress-induced social avoidance
}

Andrew L. Eagle (10 1,7, Claire E. Manning (10 1,7, Elizabeth S. Williams (1) 1, Ryan M. Bastle ${ }^{2}$, Paula A. Gajewski ${ }^{1}$, Amber Garrison ${ }^{1}$, Alexis J. Wirtz ${ }^{1}$, Seda Akguen ${ }^{1}$, Katie Brandel-Ankrapp ${ }^{1}$, Wilson Endege ${ }^{3}$, Frederick M. Boyce ${ }^{3}$, Yoshinori N. Ohnishi ${ }^{4,5}$, Michelle Mazei-Robison ${ }^{1}$, Ian Maze ${ }^{2}$, Rachel L. Neve ${ }^{6} \&$ Alfred J. Robison (i) ${ }^{1 凶}$

Chronic stress is a key risk factor for mood disorders like depression, but the stress-induced changes in brain circuit function and gene expression underlying depression symptoms are not completely understood, hindering development of novel treatments. Because of its projections to brain regions regulating reward and anxiety, the ventral hippocampus is uniquely poised to translate the experience of stress into altered brain function and pathological mood, though the cellular and molecular mechanisms of this process are not fully understood. Here, we use a novel method of circuit-specific gene editing to show that the transcription factor $\Delta$ FosB drives projection-specific activity of ventral hippocampus glutamatergic neurons causing behaviorally diverse responses to stress. We establish molecular, cellular, and circuitlevel mechanisms for depression- and anxiety-like behavior in response to stress and use circuit-specific gene expression profiling to uncover novel downstream targets as potential sites of therapeutic intervention in depression.

\footnotetext{
${ }^{1}$ Department of Physiology, Michigan State University, East Lansing, MI, USA. ${ }^{2}$ Department of Neuroscience, Icahn School of Medicine, Mount Sinai, New York, NY, USA. ${ }^{3}$ Department of Neurology, Massachusetts General Hospital, Cambridge, MA, USA. ${ }^{4}$ Department of Pharmacology, Kurume University School of Medicine, Kurume, Fukuoka, Japan. ${ }^{5}$ Department of Medical Biophysics and Radiation Biology, Faculty of Medical Sciences, Kyushu University, Fukuoka, Japan. ${ }^{6}$ Gene Technology Transfer Core, Massachusetts General Hospital, Cambridge, MA, USA. ${ }^{7}$ These authors contributed equally: Andrew L.

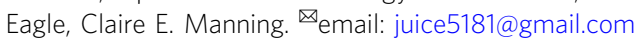


T he ventral hippocampus (vHPC) is uniquely positioned to regulate emotional responses to stress that may underlie neuropsychiatric diseases such as depression ${ }^{1,2}$. Glutamatergic vHPC neurons project to regions important in stress susceptibility and mood, including the nucleus accumbens (NAc) and basolateral amygdala (BLA), and activity of NAc-projecting vHPC neurons mediates reward behavior ${ }^{3}$ and susceptibility to stress-induced social avoidancel ${ }^{4}$. We recently demonstrated that hyperexcitability of NAc-projecting vHPC neurons also underlies female-specific susceptibility to anhedonia in the subchronic variable stress model $^{5}$, and others have recently shown that activity in this circuit is broadly predictive of stress-induced susceptibility to anxiety and social withdrawal behaviors ${ }^{6}$. However, we do not have a clear understanding of the molecular mechanisms in the vHPC driving its modulation of these target regions in depressive and anxiety disorders. Stress alters gene expression in vHPC neurons ${ }^{7-9}$, but the molecular mechanisms underlying circuit-specific vHPC gene expression and activity in response to stress are poorly understood.

$\Delta \mathrm{FosB}$ is a remarkably stable transcription factor ${ }^{10,11}$ found throughout the brain and induced by chronic neuronal activity ${ }^{12}$. $\triangle$ FosB has a well-established role in NAc in mediating stress susceptibility ${ }^{13-15}$ and regulates synaptic and intrinsic properties of NAc medium spiny neurons ${ }^{16}$. It is also necessary in dorsal hippocampus (dHPC) for learning ${ }^{17}$, and regulates the excitability of dHPC CA1 neurons ${ }^{18}$ as well as hippocampal neurogenesis ${ }^{19,20}$. $\triangle$ FosB is induced throughout HPC by stress and antidepressant treatment ${ }^{13,21-23}$, and VHPC CA $3 \Delta$ FosB is critical for the prophylactic effects of ketamine on stress responses ${ }^{23}$. This indicates that $\triangle$ FosB is a critical modulator of vHPC function and may orchestrate long-term alterations in gene expression underlying depressive and anxiety disorders. Here, we use circuitspecific CRISPR gene editing to reveal a previously unknown role for $\triangle \mathrm{FosB}$ in $\mathrm{vHPC}$ neurons projecting to NAc in resilience to social defeat stress-induced social avoidance. Furthermore, we show that $\Delta$ FosB regulates the excitability of this circuit and we identify potential downstream gene targets in this circuit that may underlie stress resilience.

\section{Results}

To investigate the induction of $\triangle \mathrm{FosB}$ in vHPC by stress, we exposed male C57Bl6/J mice to chronic social defeat stress (CSDS, Fig. S1a), which produces a variety of depressive-like symptoms, including a social avoidant phenotype in stress-susceptible mice ${ }^{24-26}$. Stress increased the number of $\Delta \mathrm{FosB}+$ dentate gyrus neurons in vHPC (Fig. S1b, c). Expanding upon our previous findings in the $\mathrm{dHPC}^{21,22}$, we found that $\triangle \mathrm{FosB}$ was also induced in all subregions of the vHPC following repeated treatment with the antidepressant fluoxetine (Fig. S1d, e), a selective serotonin reuptake inhibitor. Thus, $\triangle \mathrm{FosB}$ is induced in vHPC by both stress and antidepressants, which strongly suggests that stress-induction of $\triangle \mathrm{FosB}$ in $\mathrm{vHPC}$ is a compensatory response to counteract the effects of stress, i.e., mediating stress resilience as it does in NAc ${ }^{14}$.

Because the excitability of afferent projections from vHPC CA1 extending to NAc mediate CSDS susceptibility ${ }^{4}$ and $\triangle$ FosB regulates excitability of dHPC CA1 neurons ${ }^{18}$, we set out to investigate the role of vHPC projection neuron $\triangle \mathrm{FosB}$ in CSDS susceptibility to social avoidance. To label vHPC-NAc neurons, we utilized a mouse line expressing Cre-dependent GFP-L10a ribosomal subunit fusion protein (Rosa26 $\left.6^{e G F P-L 10 a}\right)$, and injected into NAc a persistent, retrograde viral vector expressing Cre (HSV-hEfla-Cre). Three weeks after surgery, GFP expression can be observed in ventral CA1 of vHPC (vCA1) and in ventral subiculum (vSub) (Fig. S2). Mice were then exposed to CSDS and immunostained for $\triangle \mathrm{Fos} B$. We found that CSDS induced $\triangle \mathrm{FosB}$ in labeled vHPC-NAc projections (Fig. 1a, b). To provide additional quantitative assessment, we used the same mouse model but took bilateral punches containing GFP-labeled vHPC-NAc projections and processed for circuit-specific translating ribosome affinity purification (TRAP) to enrich for actively translating Fos $B$ and $\triangle F o s B$ mRNA in vHPC-NAc neurons. CSDS produced a greater than fourfold change in these transcripts (Fig. S1f). These findings conclusively demonstrate that chronic stress increases $\triangle$ FosB expression in vHPC neurons projecting to NAc. Our next question then was to determine whether $\triangle \mathrm{FosB}$ in this circuit regulates stress phenotypes.

We first probed whether $\Delta$ FosB-mediated transcriptional regulation in vHPC is critical in resilience to stress-induced social avoidance. We virally overexpressed $\Delta J u n D$, a transcriptional inhibitor of $\triangle \mathrm{FosB}^{14}$, in either the vHPC or dHPC (Fig. 1c) and exposed mice to a subthreshold microdefeat stress (Fig. 1d) which produces a social avoidant phenotype only in mice sensitized to stress $^{24,25}$. Transcriptional inhibition of $\triangle$ FosB in vHPC reduced social interaction (SI) (Fig. 1e): $\Delta$ JunD mice spent less time interacting with a social target. Conversely, inhibition of $\triangle F o s B$ in dHPC did not produce a stress-susceptible phenotype (Fig. 1f). Furthermore, vHPC $\triangle$ FosB inhibition but did not affect baseline (unstressed) anxiety or locomotor activity (Fig. S3). Thus, $\Delta$ FosB function in vHPC is necessary for resilience to stress, but its circuit-specific role remained unknown.

Because tools to test the circuit-specific role of an individual gene have not previously been described, we developed an innovative CRISPR-Cas9 viral toolset to manipulate $\triangle \mathrm{FosB}$ expression in a circuit-specific manner (Figs. S4, S5; Suppl. Methods). Using CRISPR, we developed and screened guide RNAs (gRNA) specific to the Fos B gene, which encodes $\Delta$ FosB, and tested these in cultured mouse neuroblastoma (Neuro2A) cells. Transfection of cells with Cas9 and gRNA specific to exon I of the Fos $B$ gene reduced $\Delta$ FosB expression (Fig. S4). To confirm this in vivo, an HSV expressing both Cas9 and the gRNA was infused into dHPC of adult mice. Infected dHPC neurons had significantly reduced $\triangle$ FosB expression compared to GFP only controls (Fig. S4d, e), indicating that the CRISPR-Cas9 vector successfully mutated the Fos $B$ gene and silenced expression of $\Delta$ FosB protein (FosB KO). Previously, we have shown that $\Delta$ FosB transcriptional inhibition (via $\triangle \mathrm{JunD}$ ) in dHPC impairs learning and memory, including novel object recognition ${ }^{17}$. In keeping with our previous findings, infusion of CRISPR Fos $B \mathrm{KO}$ vector in dHPC also impaired recognition memory (Fig. S4f).

To produce a circuit-specific tool to efficiently target $\Delta$ FosB in only vHPC afferent neurons, we split the CRISPR constructs (Cas9 and gRNA) into two separate viral vectors to allow a circuit-specific intersection approach (Fig. S5a). Thus, we injected a retrograde viral vector into a projection target region and a locally expressing vector into the somatic region. Retrograde virus encoded a Cre-dependent Cas9 (HSV-hEf1a-LS1L-Cas9) and was injected into NAc; mice were allowed to recover for 3 weeks for maximal retrograde expression. We then injected into the vCA1/ vSub an HSV that locally expresses Cre and FosB gRNA (HSVIE4/5-TB-gRNA-eYFP-CMV-IRES-Cre), or control HSV (HSVIE4/5-TB-eYFP-CMV-IRES-Cre). Similar to our finding with the single-vector CRISPR strategy, the dual-vector approach specifically reduces the expression of $\triangle$ FosB in co-labeled GFP and Cas 9 vHPC cells, i.e., in NAc-projecting vHPC neurons (Fig. S5b-d).

Our tool produced a circuit-specific, CRISPR-mediated silencing of the FosB gene (FosB KO) leading to reduced $\triangle \mathrm{FosB}$ protein expression in vHPC cells projecting to NAc, allowing us to interrogate the circuit-specific effects of $\triangle$ FosB on stress susceptibility. Adult mice that received FosB KO in the vHPC-NAc circuit (Fig. 2a) were subjected to CSDS and assayed for social 

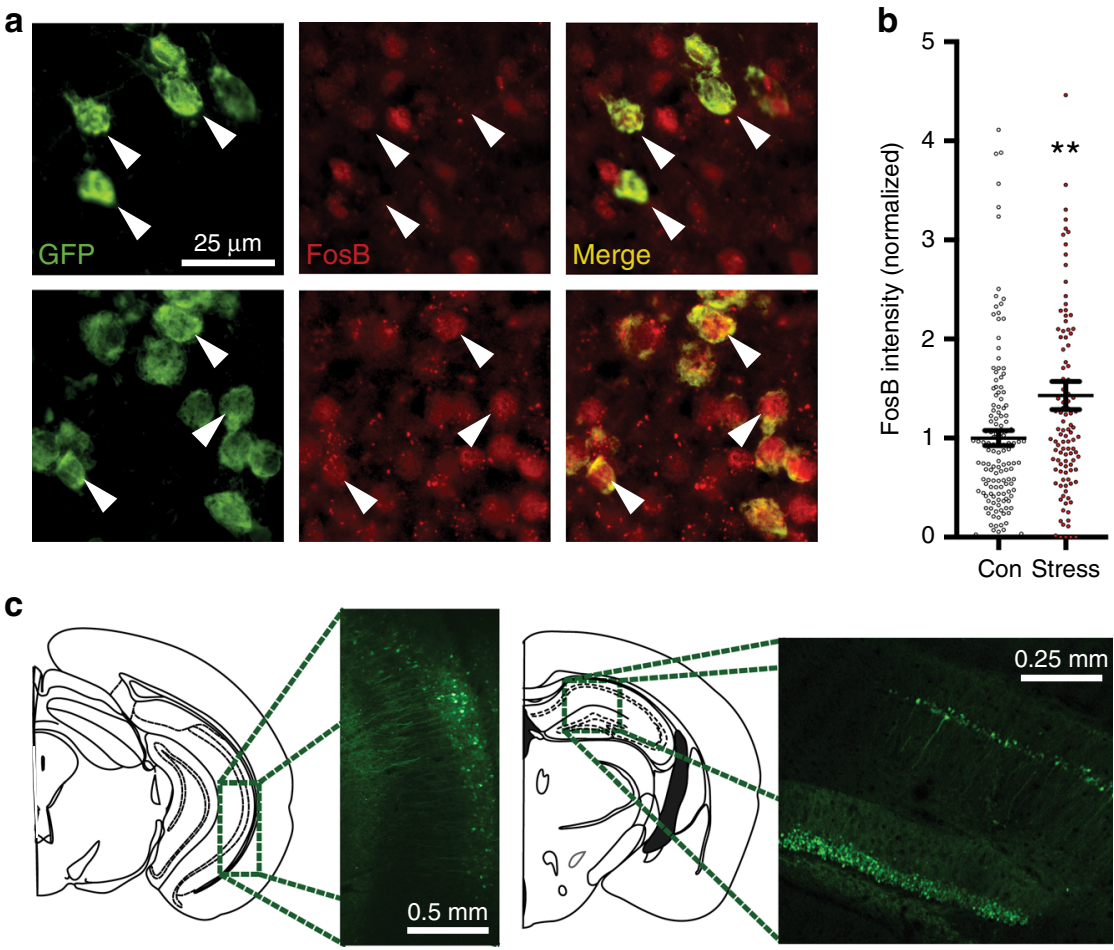

d

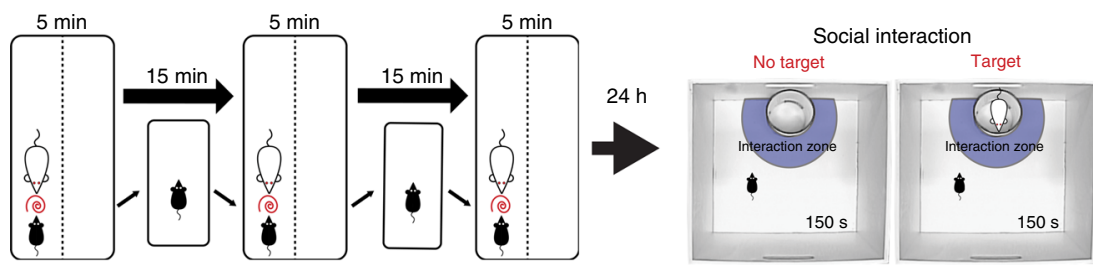

e
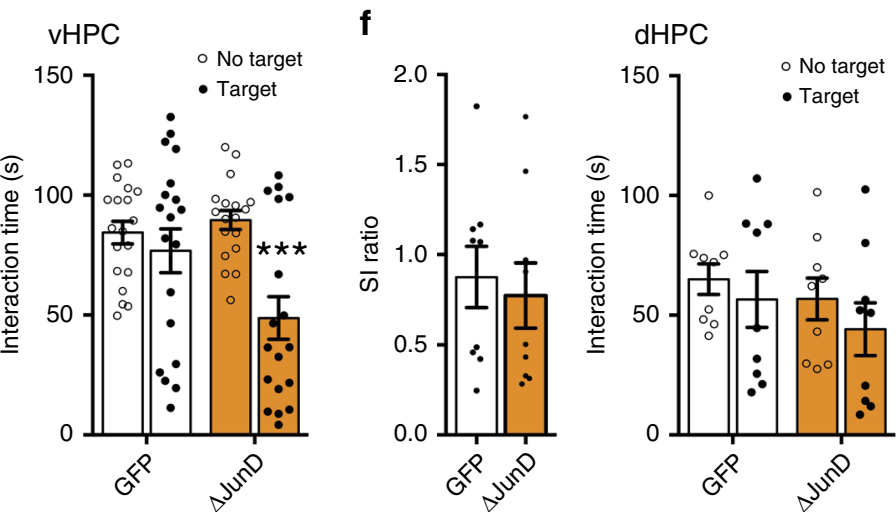

Fig. $1 \Delta$ FosB expression in the ventral hippocampus is necessary for CSDS resilience. a Representative images of vHPC CA1 coronal sections ( $\times 40)$ showing immunofluorescent labeling of NAc-projecting neurons expressing GFP (left), $\Delta$ FosB (red, middle) and merge (right). Stressed mice (bottom panels; $n=109$ cells) show increased $\Delta$ FosB signal in GFP-positive cells compared to controls (top panels; $n=147$ cells), as indicated by white arrows and quantified in (b). ${ }^{\star \star} P=0.0048$ (independent samples $t$-test compared to Control; 4 data points are outside the axis). $\mathbf{c}$ Representative figures and coronal sections showing viral-mediated GFP expression in ventral and dorsal HPC. d Experimental design for subthreshold defeat and social interaction (SI) test. e $\Delta$ JunD inhibition of $\triangle$ FosB in $\mathrm{vHPC}$ reduced SI ratio (left) and decreased investigation time of the social target (right). ${ }^{\star} P=0.0125,{ }^{\star \star \star} P<0.0001$ ( $n=19$ GFP, $n=18 \Delta$ JunD; SI ratio: independent samples $t$-test compared to GFP; investigation time: two-way mixed ANOVA with Holm-Sidak post-test GFP No Target vs Target). $\mathbf{f} \Delta \mathrm{JunD}$ expression in dHPC did not affect stress-induced social avoidance $(n=9$ mice/group). All graphs are represented as mean \pm SEM.

avoidance. FosB KO in vHPC-NAc enhanced stress-induced social avoidance (Fig. 2b) and caused a small decrease in locomotor activity (Fig. S6a). These findings suggest that $\Delta$ FosB likely regulates locomotor activity and stress susceptibility through the vHPC-NAc circuit. This is consistent with previous reports showing that vHPC afferents to NAc regulate locomotor activity $^{27,28}$. It is critical to note that this manipulation of FosB in vHPC-NAc neurons did not affect passive avoidance learning (Fig. 2c) or baseline anxiety (Fig. 2d), behaviors not associated with vHPC-NAc projections. We also observed stress effects in allodynia and anxiety, but vHPC-NAc FosB KO did not alter these behaviors (Fig. S6c-f). Therefore, $\Delta$ FosB expression in 
a

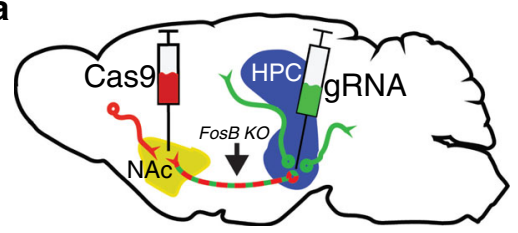

b

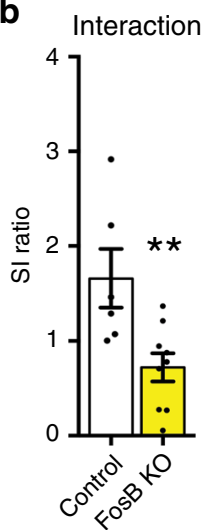

d

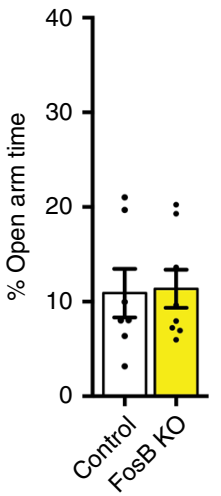

EPM

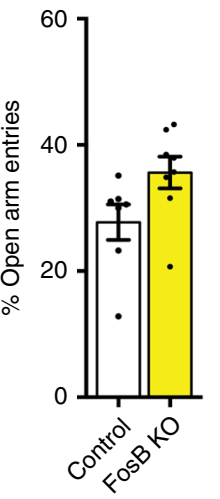

C

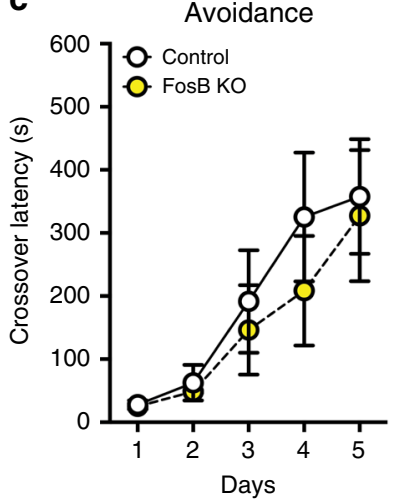

e

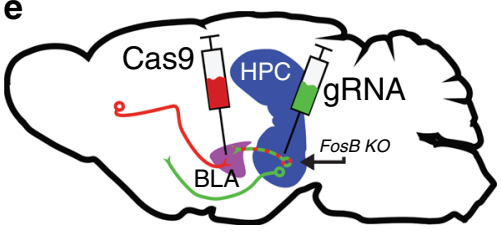

f Interaction

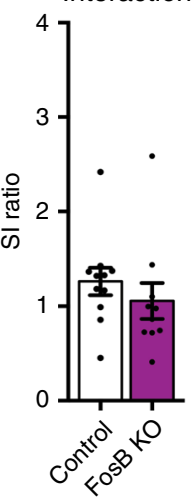

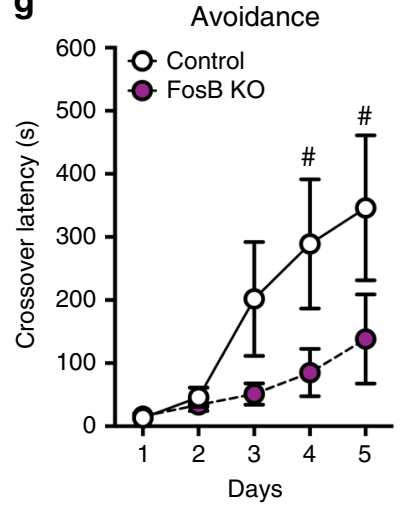

h

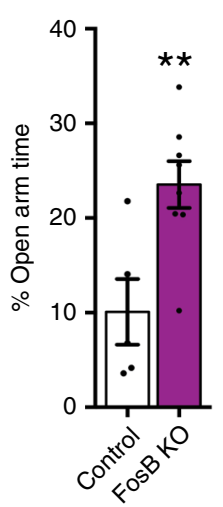

EPM

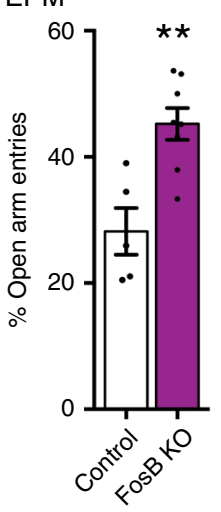

Fig. 2 Circuit-specific silencing of FosB gene in ventral hippocampal projection neurons. a Schematic of dual-vector CRISPR tool to silence FosB in vHPCNAc afferent neurons. Retrograde vector expressing Cre-dependent Cas9 (Cas9; red) is injected into NAc while local vector expressing Cre and FosB guide RNA (gRNA; green) is injected into vHPC. FosB silencing (FosB KO) occurs only in co-transduced vHPC-NAc neurons. b FosB silencing in vHPC-NAc neurons heightens stress-induced social avoidance. ${ }^{\star \star} P=0.0092$ ( $n=6$ control, $n=9$ FosB KO; independent samples $t$-test compared to control). c Fos $B$ silencing in vHPC-NAc does not affect crossover latency in the passive avoidance learning paradigm $(n=7$ control, $n=8$ FosB KO), or $\mathbf{d}$ anxiety-like behavior in the elevated plus maze (EPM). e Schematic of dual-vector CRISPR silencing FosB in vHPC-BLA neurons. $\mathbf{f}$ Fos $B$ silencing in vHPC-BLA does not affect stress-induced social avoidance ( $n=11$ control, $n=10$ FosB KO). g Fos $B$ silencing in vHPC-BLA impairs avoidance learning. $\# P=0.0614$ ( $n=6$ control, $n=8$ FosB KO; two-way mixed ANOVA with Holm-Sidak post-tests compared to control). $\mathbf{h}$ Fos $B$ silencing in vHPC-BLA decreases anxiety-like behavior in the EPM, increasing time spent and entries into the open arms. ${ }^{\star \star} P=0.0079$ for time spent and ${ }^{\star \star} P=0.0022$ for entries $(n=5$ control, $n=8$ FosB KO; independent samples t-test compared to control). All graphs are represented as mean \pm SEM.

vHPC-NAc afferents appears to be critical for a normal response to stress, and in its absence, mice are sensitized specifically to the social aspects of this stress response.

vHPC sends projections to other brain regions implicated in stress responses, including BLA, an area important in fear learning and anxiety ${ }^{29-31}$. In order to determine the circuitspecificity of our $\triangle$ FosB manipulations, we also assessed stressinduced social avoidance after FosB KO in the vHPC-BLA circuit (Fig. 2e). FosB KO in vHPC-BLA did not impair stress-induced social avoidance (Fig. 2f). Interestingly, however, it significantly impaired passive avoidance learning (Fig. 2g) and reduced anxiety-like behavior (Fig. 2h), with no change in locomotor activity (Fig. S6b). Similar observations, e.g. decreased anxiety, fear expression, and fear extinction, have been shown after vHPC lesions $2,31,32$, which suggests that $\triangle F$ osB in vHPC-BLA projections is necessary for the expression of fear and anxiety and that knockout of $\triangle$ FosB in this circuit is anxiolytic. While vHPC projections to NAc do send collaterals to other regions ${ }^{3}$, we have found that these rarely overlap and the majority of NAc and BLA projections are separate populations (Fig. S7). Taken together, these data reveal that vHPC FosB gene products regulate phenotypes in a circuit-specific manner and provide rationale for previously dissonant findings about vHPC's role in stress behaviors.

The Fos $B$ gene encodes at least 3 isoforms, including full-length FosB,$\Delta \mathrm{FosB}$, and $\Delta 2 \Delta \mathrm{FosB}$, and neuronal activity induces all of these isoforms ${ }^{12,33-35}$. It is therefore possible that any or all of these isoforms are important for the functional effects of FosB silencing in vHPC-NAc projections on stress-induced social avoidance. To investigate the specific role of $\triangle \mathrm{FosB}$ in $\mathrm{vHPC}$ projections to NAc in stress-induced behaviors, we developed an additional intersecting viral strategy that would allow us to 
a

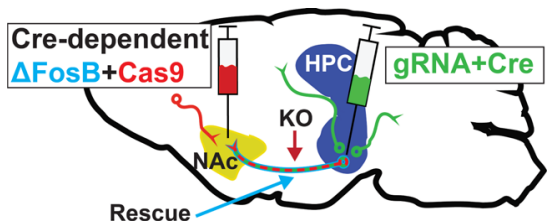

b

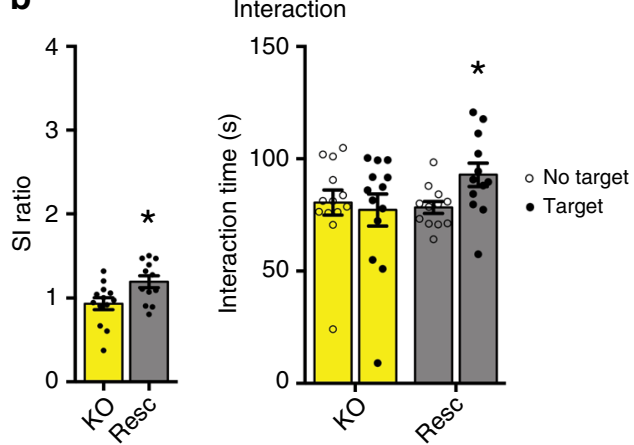

f

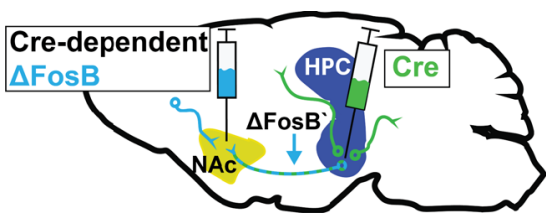

g

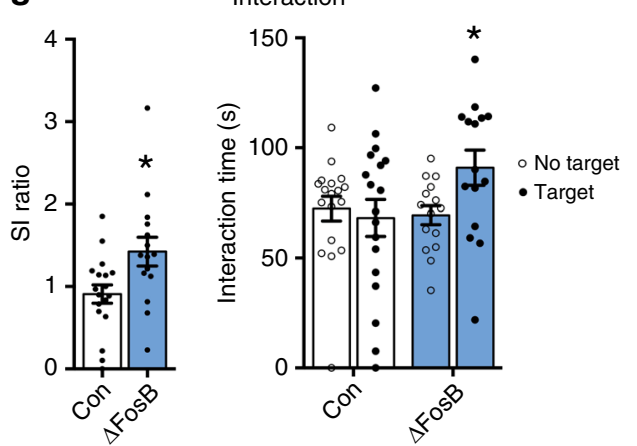

C

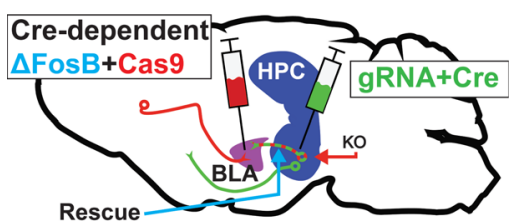

d

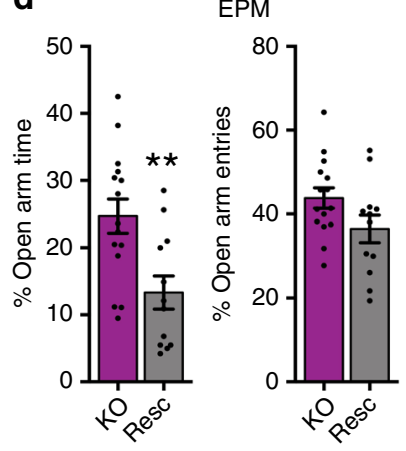

e

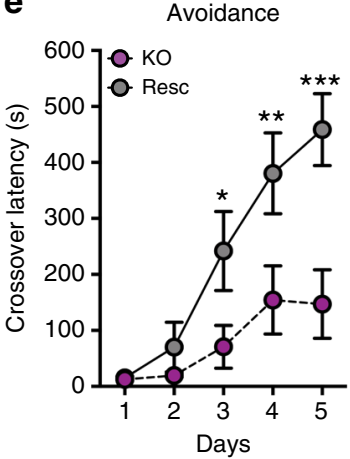

h

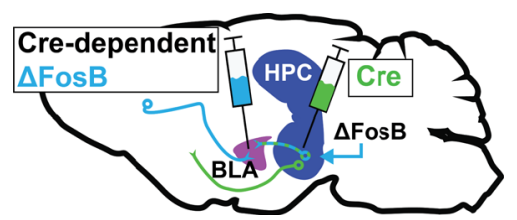

i
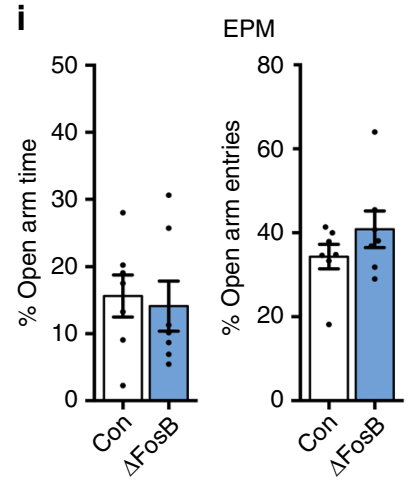

j

Avoidance

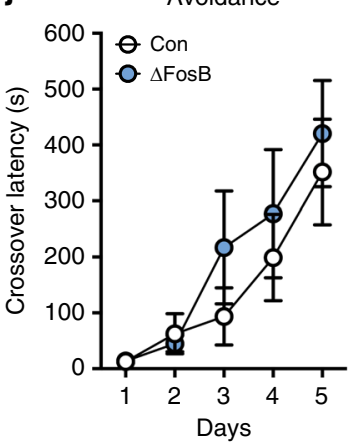

Fig. 3 Circuit-specific vHPC $\Delta$ FosB mediates stress resilience and anxiety-like behavior. a Schematic of $\triangle$ FosB rescue experiments in vHPC-NAc afferent neurons. Retrograde vector expressing Cre-dependent Cas 9 and $\triangle F o s B$ is injected into NAc while local vector expressing Cre and FosB guide gRNA is injected into $\mathrm{VHPC}$. FosB silencing (KO) occurs in co-transduced vHPC-NAc neurons, and $\triangle$ FosB overexpression is also driven by Cre in the same neurons (Rescue). b $\triangle$ FosB rescue (Resc) in vHPC-NAc neurons reverses stress-induced social avoidance, indicated by enhanced social interaction ratio (left) and increased investigation of a social target (right). ${ }^{\star} P=0.0161$ for SI ratio and ${ }^{\star} P=0.0431$ for interaction time $(n=13 \mathrm{KO}, n=12 \mathrm{Resc}$; SI ratio: independent samples $t$-test compared to KO; investigation time: two-way mixed ANOVA with Holm-Sidak post-test KO No Target vs Target). c Schematic of $\triangle$ FosB rescue experiments in $\mathrm{VHPC}$-BLA afferent neurons. $\mathbf{d} \triangle \mathrm{FosB}$ rescue in $\mathrm{vHPC}$-BLA neurons reverses the anxiolytic effects of Fos $B$ silencing, decreasing open arm time in the EPM. ${ }^{\star \star} P=0.0042(n=15 \mathrm{KO}, \mathrm{n}=12 \mathrm{Resc}$; independent samples $t$-test compared to KO). e $\Delta$ FosB rescue also reverses avoidance learning impairment. ${ }^{\star} P=0.0459$, ${ }^{\star \star} P=0.0060,{ }^{\star \star \star} P<0.0001$ ( $n=15 \mathrm{KO}, n=13 \mathrm{Resc}$; two-way mixed ANOVA with Holm-Sidak post-tests compared to KO). $\mathbf{f}$ Schematic of $\triangle \mathrm{FosB}$ overexpression in $\mathrm{VHPC}-\mathrm{NAc}$ afferent neurons. Retrograde vector expressing Cre-dependent $\Delta$ FosB is injected into $\mathrm{NAc}$ while local vector expressing $\mathrm{Cre}$ is injected into vHPC. $\triangle \mathrm{FosB}$ overexpression is driven by Cre only in co-transduced vHPC-NAc neurons ( $\triangle \mathrm{FosB}$ ). g $\triangle$ FosB overexpression in $\mathrm{VHPC}-\mathrm{NAc}$ enhances social interaction following stress, producing a resilient phenotype. ${ }^{\star} P<0.05$ ( $n=18$ control,

$n=12 \Delta$ FosB; SI ratio: independent samples $t$-test compared to control; interaction time: two-way ANOVA followed by Holm-Sidak post-test $\Delta$ FosB No Target vs Target). $\mathbf{h}$ Schematic of $\triangle$ FosB overexpression in vHPC-BLA afferent neurons. $\mathbf{i}, \mathbf{j} \Delta$ FosB overexpression in vHPC-BLA does not alter anxiety-like behavior or avoidance learning ( $n=7$ mice/group). All graphs are represented as mean \pm SEM.

overexpress recombinant $\triangle$ FosB back into the same circuit in which we use CRISPR to silence FosB gene expression (Fig. 3a). These tools cause retrograde Cre-dependent $\Delta$ FosB and Cas9 expression (Resc) or Cre-dependent Cas9 expression alone (KO). We injected these into NAc, and subsequently injected local HSV expressing Cre and the FosB gRNA into vHPC of adult mice (Fig. 3a). Critically, $\triangle$ FosB rescue in FosB KO vHPC-NAc neurons reversed the enhancement in stress-induced social avoidance
(Fig. 3b). Similarly, $\triangle$ FosB rescue in FosB KO vHPC-BLA neurons (Fig. 3c) blocked the FosB KO-mediated reduction in basal anxiety (Fig. 3d) and impairment in avoidance learning (Fig. 3e). To control for the presence of CRISPR-Cas9-mediated FosB KO, $\triangle \mathrm{FosB}$ was independently overexpressed in these same circuits by injecting retrograde Cre-inducible $\triangle \mathrm{FosB}$ in NAc or BLA (HSVhEF1 $\alpha$-LSIL- $\Delta$ FosB) and persistent Cre (AAV2-CMV-Cre-GFP) into vHPC (Fig. 3f, h), which produces $\triangle$ FosB overexpression 
specifically in vHPC-NAc-projecting cells (Fig. S8). In the absence of $F o s B$ KO, $\triangle$ FosB overexpression did not affect basal anxiety (Figs. 3i, S9a) or avoidance learning (Figs. 3j, S9b) in either projection. Interestingly, we found that overexpression of $\triangle \mathrm{FosB}$ in vHPC-NAc produced a resilient phenotype to stressinduced social avoidance (Fig. $3 \mathrm{~g}$ ). Together these data elucidate a clear role for $\triangle$ FosB in vHPC projections: $\triangle$ FosB in vHPC-NAc is necessary and sufficient for resilience to stress-induced social avoidance, and $\triangle \mathrm{FosB}$ in vHPC-BLA is necessary for the expression of fear and anxiety. Moreover, the complete behavioral rescue by $\Delta$ FosB overexpression in the same circuit in which we use the novel CRISPR system to silence the Fos $B$ gene indicates that our behavioral effects are indeed mediated by FosB knockout, and not through off-target effects of the CRISPR system. Our results demonstrate that individual genes can have disparate roles within a single brain structure based not only on heterogenous cell types but on the specific projections of the neurons in which they are expressed, which is a significant step toward illuminating the role of vHPC circuits in stress-related neuropsychiatric disease and a key finding to expand our understanding of the molecular mechanisms of complex brain functions and behaviors.

As previous findings demonstrated that increased activity in vHPC-NAc neurons underlies CSDS susceptibility ${ }^{4}$, we next sought to ascertain the role of $\triangle \mathrm{FosB}$ in the physiological properties of the vHPC-NAc circuit. We first examined the effects of $\triangle$ FosB overexpression in putative glutamatergic pyramidal neurons of vCA1 via whole-cell, patch-clamp electrophysiology in ex vivo slice preparations ${ }^{17,18}$. Viral-mediated $\triangle$ FosB overexpression reduced the excitability of vCA1 neurons, indicated by a decrease in the number of current-elicited spikes across increasing step currents (Fig. $4 \mathrm{a}, \mathrm{b}$ ) and an increase in rheobase (Fig. 4c). Little or no change was observed in most cellular properties (Table $\mathrm{S} 1$ ), but $\Delta$ FosB overexpression decreased outward rectification of the $I-V$ curve (Fig. S10a, b). Moreover, $\Delta$ FosB decreased amplitude and increased half-width of evoked action potentials and decreased the frequency of spontaneous synaptic currents (Fig. S10c-f), similar to what we have previously observed in dHPC CA1 neurons ${ }^{18}$. This suite of observations suggests that $\triangle \mathrm{FosB}$ expression reduces the excitability of vHPC neurons, likely via changes in intrinsic membrane properties. Importantly, in the vHPC-NAc circuit, stress-induced increases in $\triangle$ FosB could cause the decreased excitability previously shown to drive resilience to $\mathrm{CSDS}^{4}$, thus providing a novel molecular mechanism for this important circuit-level underpinning of stress resilience.

In order to determine whether $\triangle \mathrm{FosB}$ regulates excitability of vHPC-NAc projection neurons specifically, we crossed floxed FosB mice $\left(F o s B^{f / f l}\right)^{36}$ with the GFP-L10a line to generate floxed FosB/GFP-L10a mice (FosB KO) and non-floxed GFP-L10a littermate controls (WT). We used the same described viral retrograde Cre strategy to drive GFP-L10a expression in vHPC-NAc neurons of all mice and knock out $\triangle \mathrm{FosB}$ expression in vHPCNAc neurons of the KO, but not WT, mice (Fig. 4d-f and Fig. S11). This method is not circuit-specific (see Suppl Fig. S11c) thus making it unsuitable for assessment of stress-induced social avoidance (Suppl. Fig. S11d), yet it allows us to determine functional effects of $\triangle$ FosB KO on vHPC-NAc. Whole-cell ex vivo slice recordings from GFP-expressing vCA1-NAc neurons showed that FosB KO produces hyperexcitability in NAcprojecting vHPC neurons (Fig. $4 \mathrm{~g}-\mathrm{i}$ ). FosB $\mathrm{KO}$ increased outward rectification of the $I-V$ curve, increased amplitude and decreased half-width of evoked action potentials and increased the frequency but decreased amplitude of spontaneous synaptic currents (Fig. S12), essentially creating the opposite phenotype from $\triangle F o s B$ overexpression (Figs. $4 \mathrm{a}-\mathrm{c}$ and $\mathrm{S} 10$ ). As reduced activity in vHPC glutamatergic neurons synapsing onto NAc
MSNs confers resilience to CSDS and increased activity enhances susceptibility $^{4}$, and our results above show that vHPC-NAc $\triangle \mathrm{FosB}$ is necessary for CSDS resilience, this work supports a model in which stress-induced $\triangle$ FosB promotes hypoexcitability of NAc-projecting vHPC neurons to drive resilience to CSDSinduced social avoidance.

In order to uncover potential mechanisms by which $\Delta$ FosB regulates vHPC-NAc excitability and related behaviors, we interrogated gene expression regulated by $\triangle \mathrm{FosB}$ in NAcprojecting vHPC neurons using our circuit-specific TRAP strategy in WT and FosB KO L10-GFP mice (Fig. 5a). Purified vHPC-NAC specific mRNA was used to prepare cDNA libraries that were sequenced. We found that there was significant enrichment of neuron-specific mRNA (like kalrn) in our TRAP samples compared to input controls, with no change in enrichment of genes common to all cells (like actin; Fig. S13), indicating that our TRAP technique successfully isolated mRNA from neurons. Compared to mRNA purified from WT controls, mRNA from floxed FosB mice showed a nearly complete absence of FosB gene expression (Fig. 5b), indicating that our purified mRNA comes from vHPC neurons projecting to NAc, as retrograde viral infection in NAc is the only source of Cre in these animals. When we compared mRNAs differentially enriched in WT vs floxed FosB vHPC-NAc neurons, we uncovered hundreds of potential $\triangle$ FosB gene targets (Tables S2 and S3). In order to begin validation of some of the most interesting potential targets, we overexpressed $\triangle$ FosB in Neuro2a cells and used qPCR to assess expression of target genes (Fig. $5 \mathrm{c}$ ). $\Delta$ FosB overexpression in cell culture regulated a number of genes identified in the vHPC-NAc TRAP-Seq, including Nefm and Prkcb. We chose to examine Adra2a, which encodes the $\alpha 2 \mathrm{a}$ adrenergic receptor (a2AAR), a gene upregulated in the vHPC-NAc TRAP (Fig. S13) to highlight our approach to possible interrogation of downstream targets. Norepinephrine is associated with stress-related diseases such as depression $^{37}$, as are $\alpha 2 \mathrm{AARs}^{38,39}$, and a2AAR density is increased in the hippocampus of depressed patients ${ }^{38,40}$ and mediates the effects of antidepressants ${ }^{41,42}$. Critically, a2AARs enhance the excitability of pyramidal neurons ${ }^{43}$. Therefore, it provided a potentially viable target that may underlie $\Delta$ FosB's effects in this circuit. We found that Adra2a mRNA is downregulated in Neuro2a cells after $\triangle$ FosB overexpression (Fig. 5c), indicating that $\triangle F$ FosB represses the Adra2a gene, as it represses other genes in $\mathrm{dHPC}^{44,45}$. In order to confirm this regulation, Neuro2a cells were transfected with Cas 9 and FosB gRNA or the $\triangle$ FosB transcriptional repressor $\triangle \mathrm{JunD}$, and both conditions caused an increase in Adra $2 a$ mRNA (Fig. 5d), confirming that $\Delta$ FosB represses this gene in cultured cells. To validate this observation in vHPC-NAc neurons in vivo, we injected retrograde Cre in NAc of our GFP reporter animals and immunostained for a2AAR and GFP in both WT and floxed FosB vHPC-NAc neurons. We found that FosB KO increased the expression of a2AAR in vHPC-NAc projections (Fig. 5e, f), confirming that $\triangle$ FosB directly downregulates $A d r a 2 a$ in NAc-projecting vHPC neurons. Thus $\triangle$ FosBdependent repression of a2AAR expression in vHPC-NAc could potentially be a mechanism underlying $\triangle \mathrm{FosB}$ effects. However, considering $\triangle$ FosB also regulates a variety of gene targets, further investigation into Adra2a and many other genes is warranted.

\section{Discussion}

The current findings indicate that $\triangle \mathrm{FosB}$ is induced in response to stress in glutamatergic vHPC neurons projecting to NAc, altering gene expression to reduce circuit excitability and drive resilience to stress-induced social avoidance (Fig. 5g). As we observed that $\triangle \mathrm{FosB}$ alters synaptic inputs onto vHPC-NAc neurons, it is also possible that $\triangle \mathrm{FosB}$ may mediate 
a

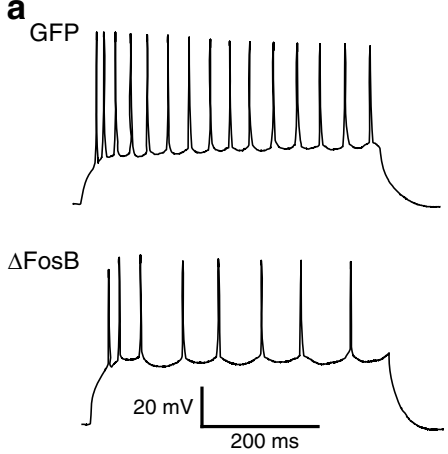

d

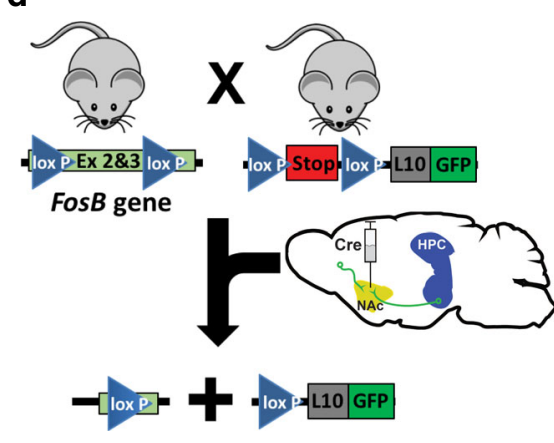

b

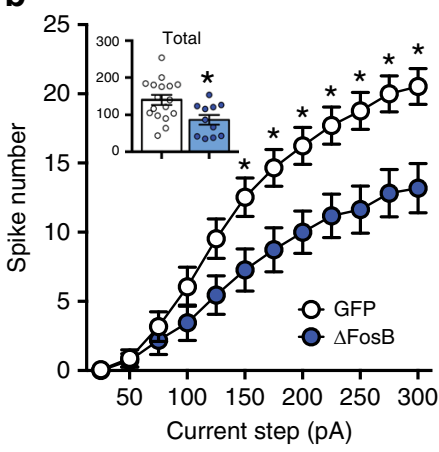

e $\quad$ WT

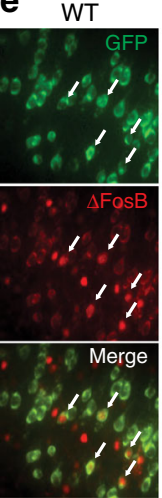

c

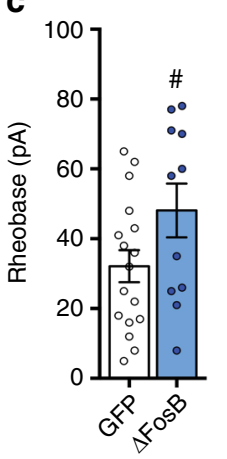

f
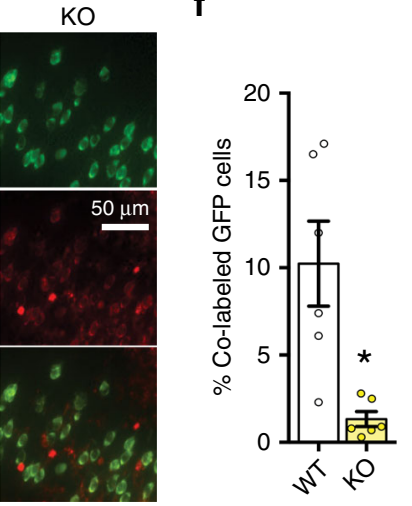

g

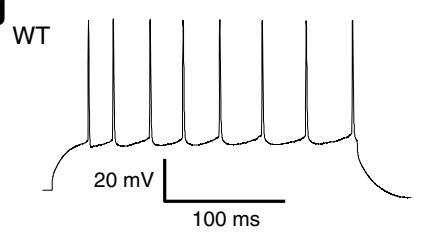

$\mathrm{KO}$

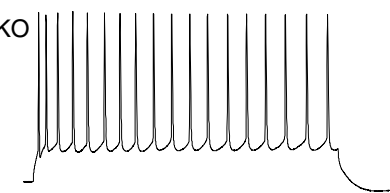

h

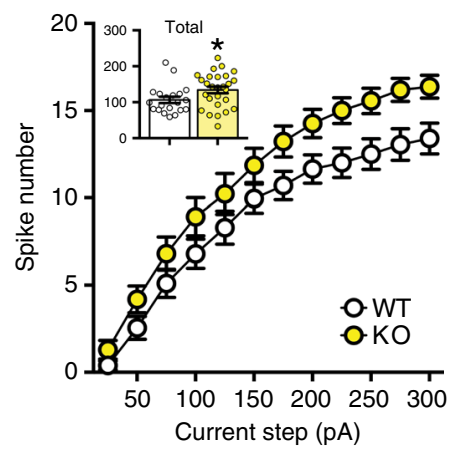

i

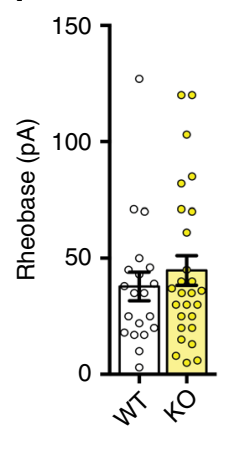

Fig. $4 \Delta$ FosB regulates the cellular excitability of NAc-projecting vHPC neurons. a Representative voltage traces from $200 \mathrm{pA}$ depolarizing current injection in ventral hippocampus CA1 (vCA1) neurons expressing GFP or GFP $+\Delta$ FosB ( $\triangle$ Fos B). $\mathbf{b} \Delta$ FosB reduces the number of elicited spikes in vCA1 neurons across increasing current steps (25-300 pA) or the total spikes for all steps (inset). ${ }^{\star} P=0.0108$ for inset; ${ }^{\star} P=0.0345$ for $150 \mathrm{pA},{ }^{\star} P=0.0135$ for $175 \mathrm{pA},{ }^{\star} P=0.0088$ for $200 \mathrm{pA},{ }^{\star} P=0.0058$ for $225 \mathrm{pA},{ }^{\star} P=0.0020$ for $250-275 \mathrm{pA}$, and ${ }^{\star} P=0.0015$ for $300 \mathrm{pA}(n=17 \mathrm{GFP}$ cells, $n=11 \Delta$ FosB cells; total: independent samples $t$-test; across steps: two-way mixed ANOVA with Holm-Sidak post-tests compared to GFP). c $\triangle F$ FosB trends toward increasing rheobase. ${ }^{\#} P=0.0680$ (independent samples $t$-test compared to GFP). d Schematic of FosB knockout in NAc-projecting neurons. Floxed Fos $B$

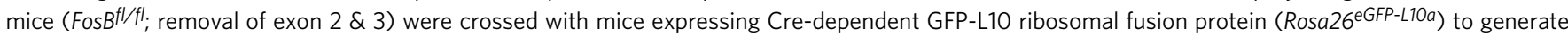
floxed FosB/GFP-L10 mice (KO) and non-floxed GFP-L10 littermate controls (WT). Retrograde Cre vector was injected in NAc to both drive GFP-L10 expression and knock out FosB in NAc-projecting neurons. e Representative coronal vHPC images ( $\times 20)$ showing NAc-projecting neurons expressing GFP (green, top), $\Delta$ FosB (red, middle), and merge (bottom). White arrows indicate co-labeled GFP $+\Delta$ FosB-expressing cells. WT (left) expressed more $\Delta$ FosB co-labeled GFP projections compared to KO quantified in (f), KO significantly reduces the \% of co-labeling of GFP $+\triangle$ FosB-expressing vHPC-NAc neurons. ${ }^{\star} P=0.0048$ ( $n=6$ mice/group; independent samples t-test compared to WT). $\mathbf{g}$ Representative voltage traces from $200 \mathrm{pA}$ depolarizing current injection in NAc-projecting GFP-labeled vCA1 neurons from WT and KO mice. $\mathbf{h}$ KO of FosB increases the number of elicited spikes in NAc-projecting vCA1 neurons across increasing current steps (25-300 pA) or the total spikes for all steps (inset). ${ }^{\star} P=0.0418$ for inset $(n=20$ WT cells, $n=27 \mathrm{KO}$ cells; total: independent samples $t$-test; across steps: two-way mixed ANOVA with Holm-Sidak post-tests versus WT). $\mathbf{i}$ No difference in rheobase was observed between WT and KO neurons ( $n=20 \mathrm{WT}$ cells, $n=27 \mathrm{KO}$ cells). All graphs are represented as mean \pm SEM.

stress-associated synaptic plasticity in vHPC. In keeping with this possibility, CSDS, like $\triangle \mathrm{FosB}$, increases the number of thin immature dendritic spines on dorsal hippocampal CA1 neurons ${ }^{17,46}$, and decreases AMPA receptor subunit levels at the postsynaptic density ${ }^{46}$. Indeed, many postsynaptic structural/ signaling proteins were uncovered in our screen of vHPC-NAc
$\Delta$ FosB gene targets (Tables S2 and S3), and future studies will use these data to explore the mechanisms by which stress alters both intrinsic excitability and synaptic inputs onto the vHPC-NAc and vHPC-BLA circuits. Ventral hippocampal circuits are key regulators of a variety of emotionally driven behaviors and hold promise for future treatments of neuropsychiatric disease if we 
a
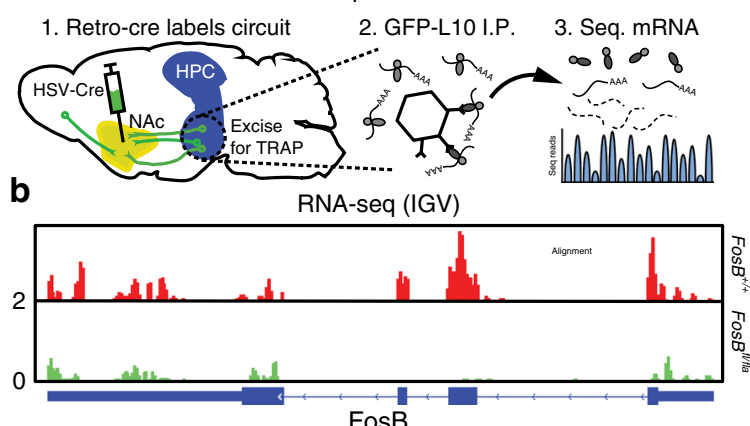

c

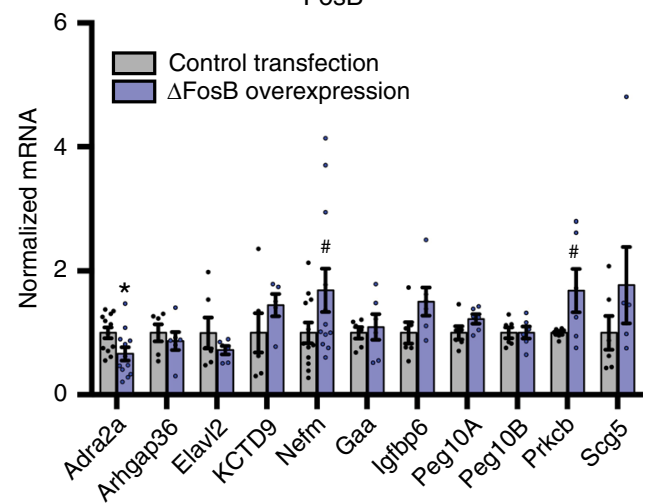

g

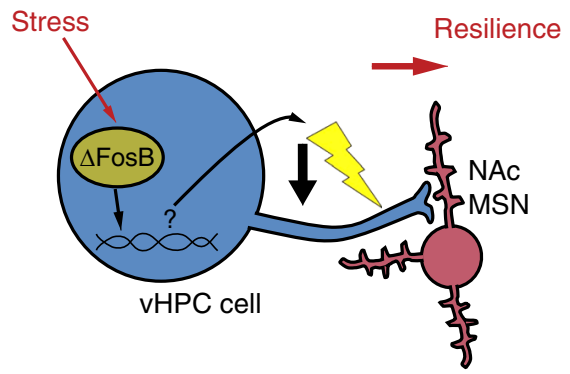

Stress Susceptibility
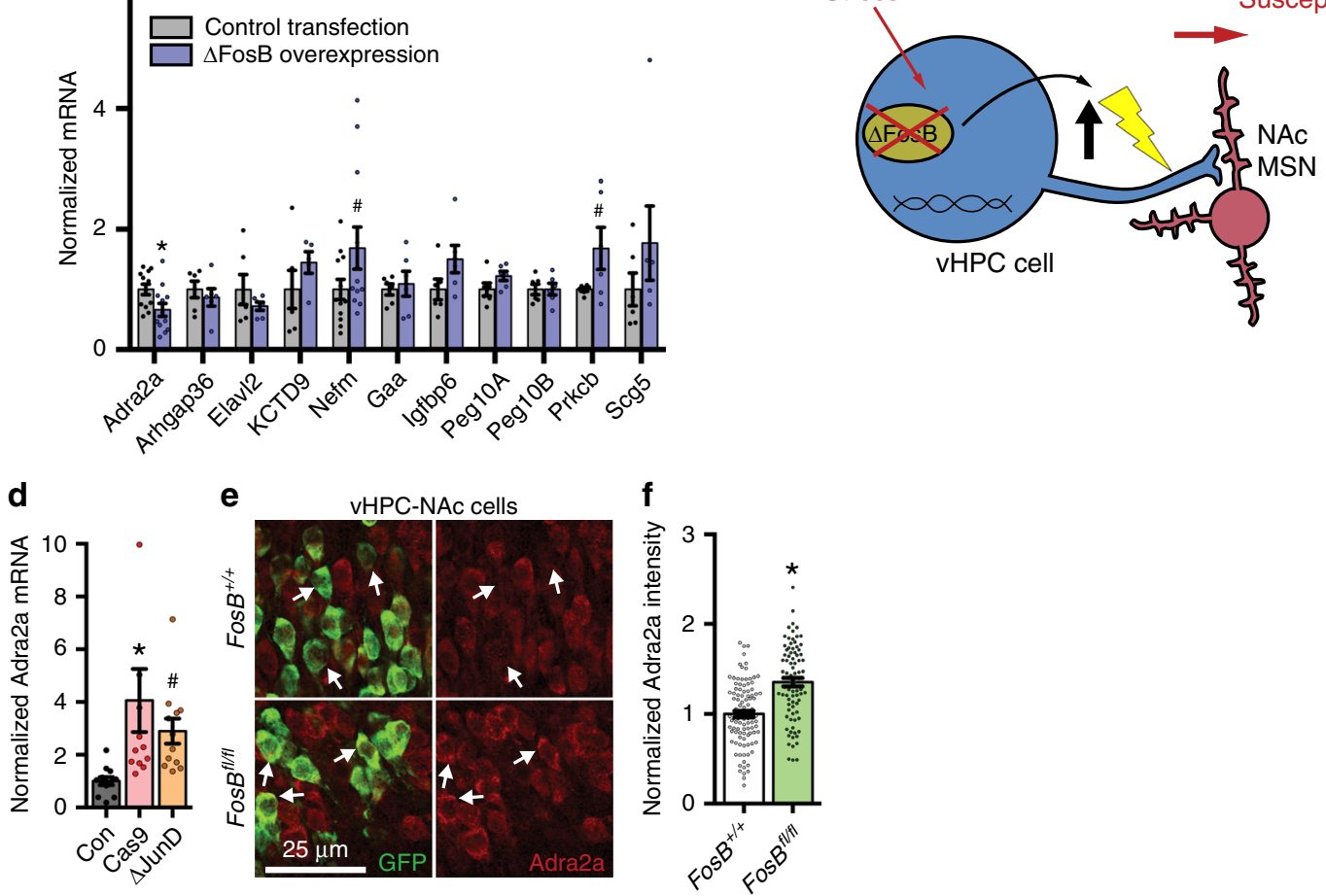

Fig. $5 \Delta$ FosB orchestrates gene expression in NAc-projecting vHPC neurons. a Experimental design for circuit-specific TRAP. Retrograde Cre vector is injected into NAc and VHPC is harvested for immunoprecipitation of ribosomes from L10-GFP-expressing NAc-projecting cells and sequencing of actively translating mRNA. b Sequencing reads (red or green peaks) for Fos $B$ gene (exons indicated by thick blue bars beneath graph) from WT (FosB ${ }^{+/+}$) and Fos $B$ $\mathrm{KO}\left(\right.$ Fos $\left.B^{f l / f l}\right)$ vHPC-NAc neuron mRNA. c $\triangle$ FosB overexpression in Neuro2A cells regulates mRNA expression of some TRAP-identified target genes. ${ }^{\star} P=0.0240$ for Adra2a, ${ }^{\# P}=0.0914$ for Nefm, ${ }^{\# P}=0.0815$ for Prkcb (independent samples $t$-test compared to Control Transfection; Adra2a $n=12$ plates/ group; Arhgap36 n=6; Elavl2 $n=6 ;$ Kctd9 $n=5-6 ;$ Nefm $n=12 ;$ Gaa $n=6$; Igfbp6 $n=6 ;$ Peg10a $n=6 ;$ Peg10b $n=6 ;$ Prkcb $n=6 ;$ Scg5 $n=6)$. d Adra2a mRNA expression is increased in Neuro2A cells transfected with $\Delta$ JunD or FosB-specific gRNA and Cas9 (Cas9). ${ }^{\star} P=0.0133,{ }^{\#} P=0.0822(n=12$ wells/ group; one-way ANOVA with Holm-Sidak post-tests versus control). e Representative coronal vHPC images (20X) showing NAc-projecting neurons expressing GFP (green) and $\alpha 2 A A R$ (red) in floxed FosB KO (Fos $B^{f l / f l}$; bottom panels) and WT (Fos $B^{+/+}$; top panels) mice. f Intensity of $\alpha 2 A A R$ expression in NAc-projecting GFP-labeled is decreased by FosB KO (Fos $\left.B^{f l / f l}\right)$. ${ }^{\star} P<0.0001\left(n=100 F_{0 s} B^{+/+}\right.$cells, $n=83$ FosB $B^{f / f l}$ cells; independent samples $t$-test compared to Fos $B^{+/+}$). e Proposed model: in resilient mice, stress strongly induces $\triangle F o s B$ in vHPC-NAc neurons initiating gene expression that underlies a protective decrease in excitability of the projection; in susceptible mice, a lack of Fos $B$ expression allows stress-associated differential gene expression to keep vHPC-NAc excitability high, driving social avoidance. All graphs are represented as mean \pm SEM.

can reveal the molecular mechanisms of their regulation. In keeping with this, recent clinical studies have demonstrated that coherence of the hippocampal-amygdala circuit correlates with anxiety phenotypes in patients ${ }^{47}$. However, the mechanisms for differences in circuit coherence cannot yet be explored in humans, emphasizing the need for preclinical models and circuitbased tools for uncovering molecular mechanisms that could yield potential treatments. Although it is possible that drugs targeting $\triangle$ FosB could be developed, we have adopted the strategy that $\triangle \mathrm{FosB}$ acts as an orchestrator of disease-relevant changes in gene expression, and thus uncovering the downstream targets of
$\Delta$ FosB transcriptional regulation may reveal myriad druggable pathways for the systemic or circuit-specific treatment of depression, potentially for symptoms of social withdrawal.

\section{Methods}

Animals. All experiments were approved by the Institutional Animal Care and Use Committee at Michigan State University in accordance with AAALAC. Male C57Bl/6J mice (3-5/cage, 7-8-week old upon arrival from Jackson Labs) were allowed at least $5 \mathrm{~d}$ to acclimate to the facility prior to any experimental procedures. The floxed FosB mouse strain $\left(\mathrm{FosB}^{\mathrm{fl} / \mathrm{fl})^{36}}\right.$ was a generous gift from the laboratory of Dr. Eric Nestler at the Icahn School of Medicine at Mount Sinai, and the Rosa26 $6^{\mathrm{eGFP}-\mathrm{L} 10 \mathrm{a}}$ mice ${ }^{48}$ were a generous gift from the laboratory of Dr. Gina 
Leinninger at Michigan State University. Male CD1 retired breeder mice (1/cage; age varies upon arrival from Charles River) were allowed at least 1 week, but more commonly $2-3$ weeks, to acclimate to the facility prior to any experimental procedures. Prior to CSDS experiments, CD1 was allowed 5-7 d to acclimate to the novel housing conditions described below. Unless otherwise stated, all mice were group housed in a 12:12 h light/dark cycle with ad libitum food and water. Temperature $\left(22^{\circ} \mathrm{C}\right)$ and humidity $(50-55 \%)$ were held constant in animal housing and behavioral testing rooms.

Surgery and viral vectors. Stereotaxic surgery was conducted as previously described $^{17}$. For $\Delta$ JunD experiments, viral vectors (AAV2-CMV-GFP or AAV2-CMV- $\triangle$ JunD-GFP) were bilaterally infused at two sites in the dHPC $\left(-2.2 \mathrm{AP}, \pm 2.0 \mathrm{ML},-2.1\right.$ and $-1.9 \mathrm{DV}$ relative to bregma, $10^{\circ}$ angle) or vHPC $\left(-3.6 \mathrm{AP}, \pm 3.2 \mathrm{ML},-4.8\right.$ and $-3.0 \mathrm{DV}$ relative to bregma, $5^{\circ}$ angle; $0.3 \mu \mathrm{L}$ per DV site) to ensure spread of transduction throughout each structure. Experimental procedures commenced at least 4 weeks following surgery. For dual virus CRISPR/Cas9 Fos $B$ gene silencing experiments, Cas9-expressing retrograde vector (HSV-hEflaLS1L-myc-Cas9; $0.5 \mu \mathrm{L})$ was infused into NAc $(+1.6 \mathrm{AP}, \pm 1.5 \mathrm{ML},-4.4 \mathrm{DV}$ relative to bregma, $10^{\circ}$ angle) or BLA ( $-1.6 \mathrm{AP}, \pm 3.4 \mathrm{ML},-4.5 \mathrm{DV}$ relative to bregma, $0^{\circ}$ angle). After 3 weeks, control viral vector (HSV-IE4/5-TB-eYFP-CMV-IRES-Cre) or FosB gRNA (HSV-IE4/5-TB-FosB gRNA-CMV-eYFP-IRES-CRE) were infused into the ventral CAl region of vHPC ( $\mathrm{vCA1} ;-3.4 \mathrm{AP}, \pm 3.2 \mathrm{ML},-4.8 \mathrm{DV}$ relative to bregma, $3^{\circ}$ angle; $0.5 \mu \mathrm{L}$ ). Experimental procedures commenced at least 2 weeks following vHPC surgeries. For rescue experiment, Cre-dependent Cas9 and $\triangle F o s B-$ expressing vector (HSV-hEF1 $\alpha$-LSIL- $\triangle$ FosB-myc-Cas9; or Cre-dependent Cas 9 alone vector as a control) was infused into NAc $(0.5 \mu \mathrm{L})$, and following 3 weeks FosB gRNA vector was infused into vCA1 $(0.5 \mu \mathrm{L})$. For $\Delta$ FosB overexpression in hippocampal circuits, Cre-dependent $\Delta$ FosB vector (HSV-hEF1 $\alpha$-LSIL- $\Delta$ FosB-IRES-GFP; $0.5 \mu \mathrm{L}$ ) was infused into NAc or BLA and Cre vector (AAV2-CMV-Cre-GFP; or AAV2CMV GFP as a control) was infused into vCA1. Experimental procedures commenced at least 4 weeks following surgeries. For electrophysiology of $\Delta$ FosB expressing vHPC neurons, viral vectors (HSV-IE4/5- $\triangle$ FosB-CMV-GFP; or HSVCMV-GFP as control) were bilateral infused into $\mathrm{vCA} 1(0.5 \mu \mathrm{L})$ and experimental procedures commenced 2-4 d following surgery. For electrophysiology of FosB KO in vHPC-NAc neurons, Cre expressing retrograde vector (HSV-hEfla-Cre) was bilaterally infused into NAc $(0.5 \mu \mathrm{L})$ of Rosa26 ${ }^{e G F P / L 10}$ mice and experimental procedures commenced at least 3 weeks following surgery. AAV viral vectors were obtained from the University of North Carolina at Chapel Hill (UNC Vector Core: https://www.med. unc.edu/genetherapy/vectorcore/) and HSV viral vectors came from Massachusetts General Hospital (Dr. Rachael Neve, Gene Delivery Technology Core: https:// researchcores.partners.org/mvvc/about).

Chronic social defeat stress. CSDS was performed as previously described ${ }^{21,24-26}$. In brief, mice were placed into the homecage of an aggressive retired breeder CD1 mouse containing a perforated plexiglass divider placed between the walls of the cage. The experimental mice were allowed to physically interact with the CD1 for $10 \mathrm{~min}$ Following the aggressive encounter, the mice were placed into the other side of the divider from the CD1 aggressor mouse allowing sensory, but not physical, contact for $24 \mathrm{~h}$. This protocol was repeated daily for $10 \mathrm{~d}$ with a new aggressor every day. Behavioral testing began the day following the final day of stress.

Subchronic defeat stress. Also called a microdefeat, subchronic defeat is an abbreviated social defeat stress protocol ${ }^{24,25}$. Mice were placed in the homecage of an aggressive retired breeder CD1 mouse and allowed to interact for 3-5 min, then removed and allowed to rest in their homecage for $15 \mathrm{~min}$ This was repeated for a total of 3 consecutive encounters in a single day. Behavioral testing began the following day.

Behavioral testing. Behavior was collected using a IR-CCD camera (Panasonic) and analyzed using automated videotracking software (CleverSys). Animals were transported to the behavioral testing rooms in their home cages and allowed $30 \mathrm{~min}$ to habituate to the room.

Social interaction. SI testing was conducted as previously described ${ }^{24,26}$. Briefly, under red light conditions, mice were placed into the center of a custom-made square, opaque arena $(38 \mathrm{~cm} W \times 38 \mathrm{~cm} L \times 35 \mathrm{~cm} \mathrm{H})$ containing an empty wire mesh cage $(10-\mathrm{cm}$ diameter) against one wall and allowed to explore for $150 \mathrm{~s}$. The experimental mice were then removed from the arena and a novel CD1 mouse was placed in the wire mesh cage. Experimental mice were then reintroduced to the arena and allowed to explore for another $150 \mathrm{~s}$. The time spent in proximity $(7.5 \mathrm{~cm})$ of the wire mesh object was defined as "interaction zone" time while the time spent in two corners $(9 \times 9 \mathrm{~cm}$ square) farthest from the object were defined as corner zone time. SI ratio was determined by calculating the time spent in the interaction zone when the $\mathrm{CD} 1$ was present divided by the time spent in the interaction zone when the $\mathrm{CD} 1$ was absent.

Elevated plus maze (EPM). Testing in EPM was conducted as previously described ${ }^{17}$. Briefly, under red light conditions, mice were placed onto the center of an EPM, with two open arms and two closed arms, and allowed to explore for $5 \mathrm{~min}$. The time spent on the open arms and the number of open arm entries were recorded.

Temporally dissociative passive avoidance (TDPA). TDPA testing was conducted as previously described ${ }^{17,49}$. Briefly, mice were placed into the lit side of light dark box. After $2 \mathrm{~min}$ of exploration, a door allowing entry into the dark side was raised. Upon entry (full body, excluding tail) into the dark side, the door was lowered and, after $5 \mathrm{~min}$, mice received a mild footshock $(0.7 \mathrm{~mA}, 2 \mathrm{~s})$. Mice were returned to their homecage after $30 \mathrm{~s}$. Testing (all of the same conditions including footshock) was repeated daily for $5 \mathrm{~d}$. The latency (s) to "cross over" from the light side to dark side was manually recorded daily.

Open field (OF). Testing in OF was conducted as previously described ${ }^{17}$. Briefly, under red light conditions, mice were placed into the center of white opaque, square custom-made $\mathrm{OF}$ and allowed to explore for $1 \mathrm{~h}$. Time spent in the center zone (50\% of the size of OF, centered) and the total distance moved (in $\mathrm{cm}$ ) were recorded.

von Frey test for tactile allodynia. Tactile allodynia was assessed using calibrated von Frey filaments as previously described ${ }^{50}$. Mice were habituated to clear plexiglass containers over a mesh floor for $1 \mathrm{~h}$. The next day mice were placed back into the containers for $30 \mathrm{~min}$ prior to testing. A series of von Frey filaments were applied to the plantar surface of the hindpaw with sufficient force to bend the filaments for at least $6 \mathrm{~s}$. A paw withdrawal or rapid flinch response was recorded. In the absence of this response, a filament of the next greater force was applied. If a response occurred, the next lower filament was applied. Paw withdrawal threshold (g) was recorded as the force that produced a $50 \%$ likelihood of withdrawal.

Novelty-suppressed feeding. Mice were restricted from ad libitum chow for $24 \mathrm{~h}$ prior to testing. Mice were placed into the corner of an OF arena (see above) with one specific difference: a single chow pellet was placed into the center under light conditions. All mice were naive to the arena. Latency to feed (s) was visually recorded as a measure of novelty-induced suppression of feeding.

Single-trial contextual avoidance. Contextual avoidance after a single conditioning trial was assessed under the same conditions as TDPA with one exception: mice were shocked immediately ( $1 \mathrm{~s}$ ) after crossover into the dark side of the box. Crossover latency was manually recorded $24 \mathrm{~h}$ later.

Tail suspension test. Mice were hung using standard laboratory tape from their tails. Tape was adhered to a horizontal bar. Mice were allowed to hang for $10 \mathrm{~min}$ Any mouse that crawled back up its tail was removed from the analysis. Immobility, defined as lack of skeletal movement for at least $1 \mathrm{~s}$, was recorded for the duration of the $10 \mathrm{~min}$ via automated tracking software (FreezeScan, CleverSys, Inc.)

Immunofluorescent staining for FosB-immunoreactivity. Rosa26 $6^{G F P / L 10 a}$ mice underwent stereotaxic surgery to infuse retrograde HSV-hEf1a-Cre into the NAc (see above). After waiting three weeks for full expression, mice were transcardially perfused with cold PBS, followed by $10 \%$ formalin. In other experiments, mice received $\triangle F$ FosB overexpression (see above) and were sacrificed and perfused 4-8 weeks following surgery. Brains from all immunostaining experiments were postfixed $24 \mathrm{~h}$ in $10 \%$ formalin, cryopreserved in $30 \%$ sucrose, and sliced frozen on an SM2010R microtome (Leica) into $35-\mu \mathrm{m}$ sections. Immunohistochemistry was performed using primary antibodies against FosB (ab11959; 1:1000; Abcam), GFP (ab5450; 1:1000; Abcam), and a2AAR (PA1-048; 1:1000; Invitrogen), and secondary antibodies (1:200; Jackson Immunoresearch) conjugated to fluorescent markers (AlexaFluor 488; Cy3; Cy5). Fluorescent images were visualized on an Olympus FluoView 1000 filter-based laser scanning confocal microscope. Intensity of a2AAR signal in individual cells was quantified using ImageJ software by an experimenter blinded to conditions.

Translational ribosomal affinity purification (TRAP) and CDNA library preparation. Three weeks following injection of retrograde HSV-Cre into NAc, Credependent L10-GFP-expressing mice (Rosa26 $6^{e G F P / L 10 a}$ ) were sacrificed and brains were immediately dissected into $1-\mathrm{mm}$ coronal sections. Transduced tissue from ventral hippocampi (vHPC) of both wild-type and FosB $B^{f / f l}$ mice was collected using 14-gauge biopsy punches guided by a fluorescent dissecting microscope (Leica) and stored at $-80^{\circ} \mathrm{C}$ until processing $(n=3$ /group, 3-4 mice pooled per $n)$.

Polyribosome-associated RNA was affinity purified as previously described ${ }^{51,52}$ Briefly, tissue was homogenized in ice-cold tissue-lysis buffer $(20 \mathrm{mM}$ HEPES [pH 7.4], $150 \mathrm{mM} \mathrm{KCl}, 10 \mathrm{mM} \mathrm{MgCl}, 0.5 \mathrm{mM}$ dithiothreitol, $100 \mu \mathrm{g} / \mathrm{ml}$ cycloheximide, protease inhibitors, and recombinant RNase inhibitors) using a motor-driven Teflon glass homogenizer. Homogenates were centrifuged for $10 \mathrm{~min}$ at $2000 \times \mathrm{g}$ $\left(4^{\circ} \mathrm{C}\right.$ ), supernatant was supplemented with $1 \%$ NP-40 (AG Scientific, \#P1505) and $30 \mathrm{mM}$ DHPC (Avanti Polar Lipids, \#850306P), and centrifuged again for $10 \mathrm{~min}$ at $20,000 \times g\left(4{ }^{\circ} \mathrm{C}\right)$. Supernatant was collected and incubated with Streptavidin 
MyOne T1 Dynabeads (Invitrogen, \#65601) that were coated with anti-GFP antibodies (Memorial Sloan-Kettering Monoclonal Antibody Facility; clone names: Htz-GFP-19F7 and Htz-GFP-19C8, $50 \mu \mathrm{g}$ per antibody per sample) using recombinant biotinylated Protein L (Thermo Fisher Scientific, \#29997) for 16-18 h on a rotator $\left(4^{\circ} \mathrm{C}\right)$ in low salt buffer $(20 \mathrm{mM}$ HEPES [pH 7.4], $350 \mathrm{mM} \mathrm{KCl}, 1 \% \mathrm{NP}-40$, $0.5 \mathrm{mM}$ dithiothreitol, $100 \mu \mathrm{g} / \mathrm{ml}$ cycloheximide). Beads were isolated and washed with high salt buffer (20 mM HEPES [pH 7.4], $350 \mathrm{mM} \mathrm{KCl,} 1 \% \mathrm{NP}-40,0.5 \mathrm{mM}$ dithiothreitol, $100 \mu \mathrm{g} / \mathrm{ml}$ cycloheximide) and RNA was purified using the RNeasy MicroKit (Qiagen, \#74004). In order to increase yield, each RNA sample was initially passed through the Qiagen MinElute ${ }^{\text {tix }}$ column three times. Following purification, RNA was quantified using a Qubit fluorometer (Invitrogen) and RNA quality was analyzed using a 4200 Agilent Tapestation (Agilent Technologies). cDNA libraries from $5 \mathrm{ng}$ total RNA were prepared using the SMARTer ${ }^{\oplus}$ Stranded Total RNA-Seq Kit (Takara Bio USA, \#635005), according to manufacturer's instructions. cDNA libraries were pooled following Qubit measurement and TapeStation analysis, with a final concentration $\sim 7 \mathrm{nM}$.

Sequencing. Sequencing was performed at the Icahn School of Medicine at Mount Sinai Genomics Core Facility (https://icahn.mssm.edu/research/genomics/corefacility). Raw sequencing reads from mice were mapped to $\mathrm{mm} 9$ using TopHat ${ }^{53}$. Counts of reads mapping to genes were obtained using HTSseq-counts software ${ }^{54}$ against Gencode vM1 (mm9) annotation. Differential expression was done using the DESeq2 package ${ }^{55}$.

Electrophysiology. Whole-cell, ex vivo slice electrophysiology was conducted as previously described ${ }^{18}$. All solutions were bubbled with $95 \% \mathrm{O}_{2}-5 \% \mathrm{CO}_{2}$ throughout the procedure. Mice were anesthetized with isoflurane anesthesia and transcardially perfused with sucrose artificial cerebrospinal fluid (aCSF; in mM: 234 sucrose, $2.5 \mathrm{KCl}, 1.25 \mathrm{NaH}_{2} \mathrm{PO}_{4}, 10 \mathrm{MgSO}_{4}, 0.5 \mathrm{CaCl}, 26 \mathrm{NaHCO}_{3}, 11$ glucose). Brains were rapidly removed, blocked, and placed in cold sucrose aCSF. Coronal sections $(250 \mu \mathrm{M})$ containing vHPC were cut on a vibratome (Leica) and transferred to an incubation chamber containing aCSF (in mM: $126 \mathrm{NaCl}, 2.5 \mathrm{KCl}$, $1.25 \mathrm{NaH}_{2} \mathrm{PO}_{4}, 2 \mathrm{MgCl}, 2 \mathrm{CaCl}, 26 \mathrm{NaHCO}_{3}, 10$ glucose) held at $34^{\circ} \mathrm{C}$ for $30 \mathrm{~min}$ before moving to aCSF at room temperature until used for recordings. Recordings were made from a submersion chamber perfused with aCSF $(2 \mathrm{~mL} / \mathrm{min})$ held at $32{ }^{\circ} \mathrm{C}$. Borosilicate glass electrodes $(3-6 \mathrm{M} \Omega$ ) were filled with $\mathrm{K}$-gluconate internal solution (in mM: 115 potassium gluconate, $20 \mathrm{KCl}, 1.5 \mathrm{MgCl}, 10$ phosphocreatineTris, 2 MgATP, $0.5 \mathrm{Na}_{3} \mathrm{GTP}$; pH 7.2-7.4; 280-285 mOsm). GFP-positive cells in the ventral CA1 region of HPC were visualized using an upright microscope (Olympus) using infrared and epifluorescent illumination. Whole-cell patch-clamp recordings were made from transfected cells using a Multiclamp 700B amplifier and Digidata 1440A digitizer (Molecular Devices) and whole-cell junction potential was not corrected. Traces were sampled $(10 \mathrm{kHz})$, filtered $(10 \mathrm{kHz})$, and digitally stored. Cells with membrane potential more positive than $-50 \mathrm{mV}$ or series resistance $>20 \mathrm{M} \Omega$ were omitted from analysis. Rheobase was measured by giving brief $(250 \mathrm{~ms})$ depolarizing $(5 \mathrm{pA})$ steps with $250 \mathrm{~ms}$ between steps. Elicited spike number was measured by issuing increasing depolarizing steps $(25-300 \mathrm{pA}$, $500 \mathrm{~ms}$ ) with $500 \mathrm{~ms}$ step intervals. For synaptic recordings of spontaneous excitatory postsynaptic currents (sEPSCs), cells were held at $-80 \mathrm{mV}$ for $2 \mathrm{~min}$ All electrophysiology recordings were made at approximately $30-32^{\circ} \mathrm{C}$ by warming the aCSF line with a single inline heater (Warner Instruments).

\section{Immunohistochemistry for detection of $\Delta$ FosB following chronic stress or} fluoxetine treatment. Immunohistochemistry was performed as previously described ${ }^{17}$, and CSDS is described above. A separate cohort of mice received oncedaily intraperitoneal injections of fluoxetine $(20 \mathrm{mg} / \mathrm{kg}$; dissolved in saline) or saline. For both experiments, mice were sacrificed $24 \mathrm{~h}$ following the last injection or defeat episode. Animals were transcardially perfused with cold PBS, followed by $10 \%$ formalin. Brains were postfixed $24 \mathrm{~h}$ in $10 \%$ formalin, cryopreserved in $30 \%$ sucrose, followed by slicing on a microtome into $35-\mu \mathrm{m}$ sections. Immunohistochemistry was performed using anti-FosB primary antibody (2251; 1:500; Cell Signaling) and biotin-conjugated secondary anti-rabbit (BA-1000; 1:1000; Vector) then visualized by $3,3^{\prime}$-diaminobenzidine staining (Vector Laboratories).

Detection of ventral hippocampal afferents to NAc. mCherry expressing retrograde vector (HSV-hEfla-mCherry) was infused into NAc and perfused coronal sections were taken at 3 weeks following surgery according to previously described protocol (see above).

CRISPR Guide RNA design and testing. gRNAs targeting exon 2 of the Fos $B$ gene were designed using e-CRISP software (www.e-CRISP.org). The top four sequences were:

\section{gRNA1: TACACCGGGAGCCGGAGTCG}

gRNA2: TTACGATCTAAAACTTACCT (this gRNA was most effective and was selected for all in vivo work described in the current manuscript; also referred to as AJR4 as it was the fourth gRNA produced for our lab)

gRNA3: TCAACATCCGCTAAGGAAGA

gRNA4: CCGTCTTCCTTAGCGGATGT
Each gRNA was tested by transfection in a mammalian expression plasmid also containing Cas9. Briefly, Neuro2a cells (N2a, American Type Culture Collection) were cultured in EMEM (ATCC) supplemented with 10\% heatinactivated fetal bovine serum (ATCC) in a $5 \% \mathrm{CO}_{2}$ humidified atmosphere at $37^{\circ} \mathrm{C}$. Cells were plated into 12 -well plates, and $24 \mathrm{~h}$ later (when cells were $\sim 30 \%$ confluent) cells were transiently transfected using Effectene (Qiagen) with a total of 200 ng DNA per well. Cells were transfected with empty vector, Cas 9 alone, or Cas 9 with a gRNA to be tested. Cells were then serum starved for $24 \mathrm{~h}$, then refed for $4 \mathrm{~h}$ to induce Fos $B$ gene expression. Cells were pelleted, samples were run on gradient polyacrylamide gels and transferred to PVDF membranes, and Western blot was performed using rabbit anti-FosB antibody (2251; 1:500; Cell Signalling) and HRP conjugated anti-rabbit secondary (PI1000; 1:40,000; Vector). Signal was detected on film and quantified using ImageJ software.

T7 endonulease surveyor analysis. T7 surveyor analysis was performed essentially as described ${ }^{56,57}$. Briefly, Neuro2a cells were plated and transfected as described above, and DNA was extracted using QuickExtract solution (Epicentre Biotechnologies). A region of the FosB gene containing the site targeted by our gRNA was amplified by PCR using the following primers:

\section{RB936: GCTTTTCCCGGAGACTACGA}

RB937: AAACCAAAGTGCAAACCGAAC

The surveyor nuclease was then used to selectively digest mismatched duplex formed from the PCR products, allowing detection of Cas9 mutated DNA.

Single virus CRISPR/Cas9 FosB KO in brain. Male C57Bl/6J mice (8-10 weeks) received surgeries in dHPC (see Methods for stereotaxic surgery above) infusing HSV expressing both Cas9 and FosB gRNA (HSV-syn-Cas9-gRNA-IRES-GFP; or HSV-CMV-GFP as a control). Mice were then tested $2 \mathrm{~d}$ following surgery for novel object recognition as previously described ${ }^{17}$. Briefly, mice were exposed for 30 min to two similar, familiar objects in an OF over 2 consecutive days. On the next day, a 5 min test for recognition was conducted, where a novel object was placed instead of one of the familiar objects. Time spent in a zone (interaction time) around the novel and familiar object was measured. Following testing, animals were sacrificed by cardiac perfusion and immunofluorescent detection of FosB and GFP expression was conducted (as above).

\section{Validation of dual virus CRISPR/Cas9 FosB KO in vHPC projections. Male} C57Bl/6J mice (8-10 weeks) received surgeries in NAc or BLA (see Methods for stereotaxic surgery above) infusing HSV expressing Cre-dependent Cas9 (see Methods for stereotaxic surgery) and 3 weeks later local HSVs expressing Cre and FosB gRNA into vHPC. Four days post gRNA viral injections, mice were sacrificed according to methods for immunohistochemistry above. Coronal sections containing vHPC were stained for Cas9 (in red; 1:500 Diagenode C15200229) and FosB (in cyan; 1:500 ab11959). FosB intensity levels for each Cas9+ neuron were measured using ImageJ, and the numbers of FosB + and FosB-Cas9-expressing neurons were quantified.

Quantitative PCR from Neuro2A cells. Neuro2A cells were treated and harvested according to prior method (see CRISPR Guide RNA design and testing), except cells were transfected with empty vector (control), $\triangle \mathrm{FosB}$, Cas9 + FosB gRNA, or $\Delta$ JunD. After cells were harvested, RNA was isolated using TriZol (Invitrogen) homogenization and chloroform layer separation. The clear RNA layer was then processed (RNAeasy MicroKit, Qiagen \#74004) and analyzed with NanoDrop. A volume of $10 \mu \mathrm{L}$ of RNA was reverse transcribed to cDNA (High Capactiy cDNA Reverse Transcription Kits Applied BioSystens \#4368814). Prior to qPCR, cDNA was diluted to $200 \mu \mathrm{L}$. The reaction mixture consisted of $10 \mu \mathrm{L}$ PowerSYBR Green PCR Master Mix (Applied Biosystems; \#436759), $2 \mu \mathrm{L}$ each of forward and reverse primers and water, and $4 \mu \mathrm{L}$ cDNA template. Samples were then heated to $95^{\circ} \mathrm{C}$ for $10 \mathrm{~min}$ (Step 1) followed by 40 cycles of $95^{\circ} \mathrm{C}$ for $15 \mathrm{~s}, 60^{\circ} \mathrm{C}$ for $15 \mathrm{~s}$, and $72^{\circ} \mathrm{C}$ for $15 \mathrm{~s}$ (Step 2), and $95^{\circ} \mathrm{C}$ for $15 \mathrm{~s}, 60^{\circ} \mathrm{C}$ for $15 \mathrm{~s}, 65^{\circ} \mathrm{C}$ for $5 \mathrm{~s}$, and $95^{\circ} \mathrm{C}$ for $5 \mathrm{~s}$ (Step 3). Analysis was carried out using the $\Delta \Delta \mathrm{C}(\mathrm{t}) \operatorname{method}^{58}$. Samples were normalized to Gapdh.

Adra2a

Forward: CAAGATCAACGACCAGAAGT

Reverse: GTCAAGGCTGATGGCGCACAG

Arhgap 36

Forward: ACTTAGAGCAGTCCTTGCGG

Reverse: GGTAGAGCTCTGTCCGGCT

Elavl2

Forward: GGTACCGCCGCCAGGAAACACAACTGTCTAATGGG Reverse: GCGGCCGCACTGAGGACAAGAGCTCATTAGGCTTTGT Gapdh

Forward: AGGTCGGTGTGAACGGATTTG

Reverse: TGTAGACAATGTAGTTGAGGTCA Igfbp6

Forward:GGTCTACAGCCCTAAGTGCG

Reverse: AGGGGCCCATCTCACTATCT 


\author{
Kctd9 \\ Forward: CGGGTCACGCTGTTCTTGA \\ Reverse: ACAGCACATCATCATCCCTGA \\ Nefm \\ Forward: CAGCTACCAGGACACCATCCAG \\ Reverse: GTGTACAGAGGCCCGGTGAT \\ Peg10A \\ Forward: CCGATACACGCGTTTCCAAC \\ Reverse: TAAAACCCGCCTGTTCCACA \\ Peg10B \\ Forward: AATCCTCGTGTGGAACAGGC \\ Reverse: TCATCATCTTCGGCGTCAGG \\ Prkcb \\ Forward: CAGAGATTGCCATCGGTCTGT \\ Reverse: CCCCTCAGAATCCAGCATCA \\ $\operatorname{Scg} 5$ \\ Forward: ATCAAGGCTACCCAGACCCT \\ Reverse: GGATTGACACTCCTCCGCTT
}

Statistics and reproducibility. For all experiments, alpha criterion was set to 0.05 . SI testing was analyzed for SI ratio and interaction zone time. SI ratio was analyzed by independent samples $t$-tests between groups. Interaction time was analyzed by mixed two-way ANOVAs with Target as the within factor. Omnibus ANOVAs were followed by Holm-Sidak corrected post hoc comparisons between groups. Avoidance learning in the TDPA was analyzed using mixed two-way ANOVAs with Days as the within factor followed by Holm-Sidak corrected post hoc com parisons between groups. EPM and OF behavior was analyzed by independent samples $t$-tests between groups. For novel object recognition experiment, object interaction time was analyzed using mixed two-way ANOVAs with Object as the within factor followed by Holm-Sidak corrected post hoc comparisons within groups. For all western blotting and immunohistochemistry results, data were analyzed by independent samples $t$-tests between groups. Spike number in electrophysiology experiments was analyzed by mixed two-way ANOVAs with Current as the within factor followed by Holm-Sidak corrected post hoc comparisons between groups. $I-V$ curves in electrophysiology experiments were analyzed by mixed two-way ANOVAs with Voltage as the within factor followed by Holm-Sidak corrected post hoc comparisons between groups. For all other electrophysiological measures: rheobase, spike amplitude, spike half-width, sEPSC amplitude, sEPSC frequency, and other cellular properties (Table S1) data were analyzed by independent samples $t$-tests between groups. Refer to Tables S4 and S5 for all omnibus statistical results.

In most cases, behavioral experiments were conducted in no less than two cohorts to ensure reproducibility. For all viral manipulation experiments, confirmation of viral targeting was conducted using antibodies to enhance native GFP signal (e.g., Fig. 1c). Data from mice lacking targeting in a brain region were removed from analyses. All representative images and electrophysiological recording traces were selected based on data representing the mean for each group. Representative micrograph displayed in Fig. 4e were reproduced in all samples ( $n=6$ /group). The same is true in Fig. 5e. FosB staining micrographs displayed in Fig. S1 were replicated; and micrographs and data shown are from a second replication. Representative micrographs in Figs. S4d, S5b, and S8a were replicated in preliminary studies (two replications for S4d; four replications for S5b; one replication for S8a) and reproduced in the final data shown. Representative micrograph shown in Fig. S11b has been replicated multiple times in our lab and published $^{5}$. In addition, all experiments using the methods proposed in Fig. S11a replicated the same pattern of expression shown in S11b.

Reporting summary. Further information on research design is available in the Nature Research Reporting Summary linked to this article.

\section{Data availability}

Sequencing datasets generated during and analyzed during the current study are available in the NIH GEO repository (https://www.ncbi.nlm.nih.gov/geo/) with the accession code GSE137283. All the other data supporting the findings of this study are available within the article and its supplementary information files and from the corresponding author upon reasonable request. A reporting summary for this article is available as a Supplementary Information file. Source data are provided with this paper.

Received: 21 January 2019; Accepted: 22 July 2020;

Published online: 08 September 2020

\section{References}

1. Fanselow, M. S. \& Dong, H.-W. Are the dorsal and ventral hippocampus functionally distinct structures? Neuron 65, 7-19 (2010).
2. Bannerman, D. M. et al. Regional dissociations within the hippocampusmemory and anxiety. Neurosci. Biobehav Rev. 28, 273-283 (2004).

3. LeGates, T. A. et al. Reward behaviour is regulated by the strength of hippocampus-nucleus accumbens synapses. Nature 564, 258-262 (2018).

4. Bagot, R. C. et al. Ventral hippocampal afferents to the nucleus accumbens regulate susceptibility to depression. Nat. Commun. 6, 7062 (2015).

5. Williams, E. S. et al. Androgen-dependent excitability of mouse ventral hippocampal afferents to nucleus accumbens underlies sex-specific susceptibility to stress. Biol. Psychiatry 87, 492-501 (2020).

6. Muir, J. et al. Ventral-hippocampal afferents to nucleus accumbens encode both latent vulnerability and stress-induced susceptibility. Biol. Psychiatry S0006-3223, 31627-31629 (2020).

7. Floriou-Servou, A. et al. Distinct proteomic, transcriptomic, and epigenetic stress responses in dorsal and ventral hippocampus. Biol. Psychiatry 84, 531-541 (2018).

8. Pacheco, A. et al. Chronic stress triggers expression of immediate early genes and differentially affects the expression of AMPA and NMDA subunits in dorsal and ventral hippocampus of rats. Front Mol. Neurosci. 10, 244 (2017).

9. Kenworthy, C. A. et al. Social defeat induces changes in histone acetylation and expression of histone modifying enzymes in the ventral hippocampus, prefrontal cortex, and dorsal raphe nucleus. Neuroscience 264, 88-98 (2014).

10. Carle, T. L. et al. Proteasome-dependent and -independent mechanisms for FosB destabilization: identification of FosB degron domains and implications for $\Delta$ FosB stability. Eur. J. Neurosci. 25, 3009-3019 (2007).

11. Ulery, P. G., Rudenko, G. \& Nestler, E. J. Regulation of $\Delta$ FosB stability by phosphorylation. J. Neurosci. 26, 5131-5142 (2006).

12. Nestler, E. J., Kelz, M. B. \& Chen, J. $\triangle$ FosB: a molecular mediator of long-term neural and behavioral plasticity. Brain Res. 835, 10-17 (1999).

13. Perrotti, L. I. et al. Induction of $\triangle \mathrm{FosB}$ in reward-related brain structures after chronic stress. J. Neurosci. 24, 10594-10602 (2004).

14. Vialou, V. et al. DeltaFosB in brain reward circuits mediates resilience to stress and antidepressant responses. Nat. Neurosci. 13, 745-752 (2010).

15. Robison, A. J. \& Nestler, E. J. Transcriptional and epigenetic mechanisms of addiction. Nat. Rev. Neurosci. 12, 623-637 (2011).

16. Grueter, B. A., Robison, A. J., Neve, R. L., Nestler, E. J. \& Malenka, R. C. FosB differentially modulates nucleus accumbens direct and indirect pathway function. Proc. Natl Acad. Sci. USA 110, 1923-1928 (2013).

17. Eagle, A. L. et al. Experience-dependent induction of hippocampal $\Delta F$ FosB controls learning. J. Neurosci. 35, 13773-13783 (2015).

18. Eagle, A. L., Williams, E. S., Beatty, J. A., Cox, C. L. \& Robison, A. J. $\Delta$ FosB decreases excitability of dorsal hippocampal CA1 neurons. eNeuro 5, ENEURO.0104-0118.2018 (2018).

19. Manning, C. E. et al. Hippocampal subgranular zone FosB expression is critical for neurogenesis and learning. Neuroscience 406, 225-233 (2019).

20. Yutsudo, N. et al. fosB-null mice display impaired adult hippocampal neurogenesis and spontaneous epilepsy with depressive behavior. Neuropsychopharmacology 38, 895-906 (2013).

21. Vialou, V. et al. Differential induction of FosB isoforms throughout the brain by fluoxetine and chronic stress. Neuropharmacology 99, 28-37 (2015).

22. Cooper, S. E. et al. Comparison of chronic physical and emotional social defeat stress effects on mesocorticolimbic circuit activation and voluntary consumption of morphine. Sci. Rep. 7, 8445 (2017).

23. Mastrodonato, A. et al. Ventral CA3 activation mediates prophylactic ketamine efficacy against stress-induced depressive-like behavior. Biol. Psychiatry 84, 846-856 (2018).

24. Krishnan, V. et al. Molecular adaptations underlying susceptibility and resistance to social defeat in brain reward regions. Cell 131, 391-404 (2007).

25. Golden, S. A., Covington, H. E., Berton, O. \& Russo, S. J. A standardized protocol for repeated social defeat stress in mice. Nat. Protoc. 6, 1183-1191 (2011).

26. Berton, O. et al. Essential role of BDNF in the mesolimbic dopamine pathway in social defeat stress. Science 311, 864-868 (2006).

27. Blaha, C. D., Yang, C. R., Floresco, S. B., Barr, A. M. \& Phillips, A. G. Stimulation of the ventral subiculum of the hippocampus evokes glutamate receptor-mediated changes in dopamine efflux in the rat nucleus accumbens. Eur. J. Neurosci. 9, 902-911 (1997).

28. Floresco, S. B., Todd, C. L. \& Grace, A. A. Glutamatergic afferents from the hippocampus to the nucleus accumbens regulate activity of ventral tegmental area dopamine neurons. J. Neurosci. 21, 4915-4922 (2001).

29. Fanselow, M. S. \& LeDoux, J. E. Why we think plasticity underlying pavlovian fear conditioning occurs in the basolateral amygdala. Neuron 23, 229-232 (1999).

30. Etkin, A. et al. Individual differences in trait anxiety predict the response of the basolateral amygdala to unconsciously processed fearful faces. Neuron 44, 1043-1055 (2004).

31. Sierra-Mercado, D., Padilla-Coreano, N. \& Quirk, G. J. Dissociable roles of prelimbic and infralimbic cortices, ventral hippocampus, and basolateral 
amygdala in the expression and extinction of conditioned fear. Neuropsychopharmacology 36, 529-538 (2011).

32. Kjelstrup, K. G. et al. Reduced fear expression after lesions of the ventral hippocampus. Proc. Natl Acad. Sci. USA 99, 10825-10830 (2002).

33. Nakabeppu, Y. \& Nathans, D. A naturally occurring truncated form of FosB that inhibits Fos/Jun transcriptional activity. Cell 64, 751-759 (1991).

34. Chen, J., Kelz, M. B., Hope, B. T., Nakabeppu, Y. \& Nestler, E. J. Chronic Fosrelated antigens: stable variants of $\triangle \mathrm{FosB}$ induced in brain by chronic treatments. J. Neurosci. 17, 4933-4941 (1997).

35. Bading, $\mathrm{H}$. et al. N-methyl-d-aspartate receptors are critical for mediating the effects of glutamate on intracellular calcium concentration and immediate early gene expression in cultured hippocampal neurons. Neuroscience $\mathbf{6 4}$, 653-664 (1995).

36. Ohnishi, Y. N. et al. Generation and validation of a floxed FosB mouse line. bioRxiv https://doi.org/10.1101/179309 (2017).

37. Goddard, A. W. et al. Current perspectives of the roles of the central norepinephrine system in anxiety and depression. Depress. Anxiety 27, 339-350 (2010).

38. Cottingham, C. \& Wang, Q. $\alpha 2$ adrenergic receptor dysregulation in depressive disorders: Implications for the neurobiology of depression and antidepressant therapy. Neurosci. Biobehav Rev. 36, 2214-2225 (2012).

39. Cottingham, C., Chen, H., Chen, Y., Peng, Y. \& Wang, Q. in Current Topics in Membranes (ed. Wang, Q.) Chapter 8 (Academic Press, 2011).

40. González, A. M. et al. Autoradiographic demonstration of increased a2adrenoceptor agonist binding iites in the hippocampus and frontal cortex of depressed suicide victims. J. Neurochem. 63, 256-265 (1994)

41. Yanpallewar, S. U. et al. a2-Adrenoceptor blockade accelerates the neurogenic, neurotrophic, and behavioral effects of chronic antidepressant treatment. J. Neurosci. 30, 1096-1109 (2010).

42. Zhang, H.-T., Whisler, L. R., Huang, Y., Xiang, Y. \& O'Donnell, J. M. Postsynaptic $\alpha-2$ adrenergic receptors are critical for the antidepressant-like effects of desipramine on behavior. Neuropsychopharmacology 34, 1067 (2008).

43. Carr, D. B., Andrews, G. D., Glen, W. B. \& Lavin, A. a2-Noradrenergic receptors activation enhances excitability and synaptic integration in rat prefrontal cortex pyramidal neurons via inhibition of $\mathrm{HCN}$ currents. J. Physiol. 584, 437-450 (2007).

44. You, J. C. et al. Genome-wide profiling reveals functional diversification of FosB gene targets in the hippocampus of an Alzheimer's disease mouse model. PLOS ONE 13, e0192508 (2018).

45. You, J. C. et al. Epigenetic suppression of hippocampal calbindin-D28k by $\triangle$ FosB drives seizure-related cognitive deficits. Nat. Med. 23, 1377 (2017).

46. Iñiguez, S. D. et al. Social defeat stress induces depression-like behavior and alters spine morphology in the hippocampus of adolescent male C57BL/6 mice. Neurobiol. Stress 5, 54-64 (2016).

47. Kirkby, L. A. et al. An amygdala-hippocampus subnetwork that encodes variation in human mood. Cell 175, 1688-1700 (2018).

48. Brown, J. A. et al. Loss of action via neurotensin-leptin receptor neurons disrupts leptin and ghrelin-mediated control of energy balance. Endocrinology 158, 1271-1288 (2017).

49. Eagle, A. L., Wang, H. \& Robison, A. J. Sensitive assessment of hippocampal learning using temporally dissociated passive avoidance task. Bio Protoc. 6, e1821 (2016)

50. Laumet, G. et al. G9a is essential for epigenetic silencing of $\mathrm{K}+$ channel genes in acute-to-chronic pain transition. Nat. Neurosci. 18, 1746-1755 (2015).

51. Heiman, M., Kulicke, R., Fenster, R. J., Greengard, P. \& Heintz, N. Cell typespecific mRNA purification by translating ribosome affinity purification (TRAP). Nat. Protoc. 9, 1282-1291 (2014).

52. Heiman, M. et al. A translational profiling approach for the molecular characterization of CNS cell types. Cell 135, 738-748 (2008).

53. Trapnell, C. et al. Differential gene and transcript expression analysis of RNAseq experiments with TopHat and Cufflinks. Nat. Protoc. 7, 562 (2012).
54. Anders, S., Pyl, P. T. \& Huber, W. HTSeq-a Python framework to work with high-throughput sequencing data. Bioinformatics 31, 166-169 (2014).

55. Love, M. I., Huber, W. \& Anders, S. Moderated estimation of fold change and dispersion for RNA-seq data with DESeq2. Genome Biol. 15, 550 (2014).

56. Vouillot, L., Thelie, A. \& Pollet, N. Comparison of T7E1 and surveyor mismatch cleavage assays to detect mutations triggered by engineered nucleases. G3 5, 407-415 (2015).

57. Guschin, D. Y. et al. in Engineered Zinc Finger Proteins: Methods and Protocols (eds Mackay, J.P. \& Segal, D.J.) (Humana Press, 2010).

58. Tsankova, N. M. et al. Sustained hippocampal chromatin regulation in a mouse model of depression and antidepressant action. Nat. Neurosci. $\mathbf{9}$, 519-525 (2006).

\section{Acknowledgements}

We would like to thank Kenneth Moon for outstanding technical assistance. This work was supported by NIMH R01 111604, NIDA R01 040621, and NINDS R01 085171 (to A.J.R.) and a NARSAD Young Investigator Award from the Brain and Behavior Research Foundation (to A.L.E.). Rosa26 $6^{\text {GFP-L10a }}$ mice were generously provided by Dr. Gina Leinninger at Michigan State University.

\section{Author contributions}

A.L.E., C.E.M., F.M.B., M.M.R., I.M., R.L.N., and A.J.R. conceived and interpreted experiments; A.L.E., C.E.M., E.S.W., R.M.B., P.A.G., A.G., A.J.W., S.A., K.B.-A., and W.E performed experiments; A.L.E. and A.J.R. wrote the paper; R.L.N. and Y.N.O. contributed tools.

\section{Competing interests}

The authors declare no competing interests.

\section{Additional information}

Supplementary information is available for this paper at https://doi.org/10.1038/s41467020-17825-x.

Correspondence and requests for materials should be addressed to A.J.R

Peer review information Nature Communications thanks the anonymous reviewers for their contribution to the peer review of this work. Peer reviewer reports are available.

Reprints and permission information is available at http://www.nature.com/reprints

Publisher's note Springer Nature remains neutral with regard to jurisdictional claims in published maps and institutional affiliations.

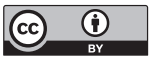

Open Access This article is licensed under a Creative Commons Attribution 4.0 International License, which permits use, sharing, adaptation, distribution and reproduction in any medium or format, as long as you give appropriate credit to the original author(s) and the source, provide a link to the Creative Commons license, and indicate if changes were made. The images or other third party material in this article are included in the article's Creative Commons license, unless indicated otherwise in a credit line to the material. If material is not included in the article's Creative Commons license and your intended use is not permitted by statutory regulation or exceeds the permitted use, you will need to obtain permission directly from the copyright holder. To view a copy of this license, visit http://creativecommons.org/ licenses/by/4.0/.

(C) The Author(s) 2020 